\title{
Third-order particle-hole ring diagrams with contact-interactions and one-pion exchange 1
}

\author{
N. Kaiser \\ Physik-Department T39, Technische Universität München, D-85748 Garching, Germany
}

\begin{abstract}
The third-order particle-hole ring diagrams are evaluated for a NN-contact interaction of the Skyrme type. The pertinent four-loop coefficients in the energy per particle $\bar{E}\left(k_{f}\right) \sim k_{f}^{5+2 n}$ are reduced to double-integrals over cubic expressions in euclidean polarization functions. Dimensional regularization of divergent integrals is performed by subtracting power-divergences and the validity of this method is checked against the known analytical results at second-order. The complete $\mathcal{O}\left(p^{2}\right) \mathrm{NN}$-contact interaction is obtained by adding two tensor terms and their third-order ring contributions are also calculated in detail. The third-order ring energy arising from long-range $1 \pi$-exchange is computed and it is found that direct and exchange contributions are all attractive. The very large size of the pion-ring energy, $\bar{E}\left(k_{f 0}\right) \simeq-92 \mathrm{MeV}$ at saturation density, is however in no way representative for that of realistic chiral NNpotentials. Moreover, the third-order (particle-particle and hole-hole) ladder diagrams are evaluated with the full $\mathcal{O}\left(p^{2}\right)$ contact interaction and the simplest three-ring contributions to the isospin-asymmetry energy $A\left(k_{f}\right) \sim k_{f}^{5}$ are studied.
\end{abstract}

\section{$1 \quad$ Introduction and summary}

Due to the progress in constructing nuclear forces within chiral effective field theory [1, 2, 3] and the advances in many-body techniques, new paths have opened to perform systematically improvable calculations of nuclear many-body systems. Infinite (isospin-symmetric) nuclear matter and pure neutron matter have been studied extensively based on chiral two- and three-nucleon lowmomentum interactions within various many-body frameworks, such as many-body perturbation theory [4, 5, 6, 7, 8], an in-medium chiral perturbation approach [9], the selfconsistent Green's function method [10, 11, the Brueckner-Hartree-Fock approach [12], and quantum Monte-Carlo simulations [13]. When including the (leading) chiral 3N-force in the form of a density-dependent effective NN-interaction, calculations at least up to second order are necessary in order to achieve reasonable saturation properties and agreement with empirical bulk quantities of nuclear matter. Several works [5, 6, 11] have also studied the importance of the third-order particle-particle and hole-hole ladder contributions, but the third-order particle-hole ring diagram is often neglected due to its more complicated momentum and spin recouplings when implementing the NN-potential in terms of partial-wave matrix-elements, which significantly increases the computational costs. In ref. [8] it was found that the third-order particle-hole ring diagram gives a contribution of about (12) $\mathrm{MeV}$ in isospin-symmetric nuclear matter at $\rho_{0} \simeq 0.16 \mathrm{fm}^{-3}$ when computed from low-resolution chiral NN-potentials. This size of the three-ring energy per particle has been confirmed recently in ref.[14], where the extensive numerical computations involving multiple partial-wave sums and momentum-space integrations have been benchmarked against semi-analytical evaluations for testinteractions of the one-boson exchange type.

The purpose of the present paper is to calculate the third-order particle-hole ring diagrams for relatively simple two-body interactions that just make feasible an analytical treatment. A suitable candidate for such an interesting and worthwhile exploration within many-body perturbation theory is the NN-contact interaction of the Skyrme type. The latter is widely and successfully

\footnotetext{
${ }^{1}$ This work has been supported in part by DFG and NSFC (CRC110).
} 
used for non-relativistic nuclear structure calculations of medium-mass and heavy nuclei [15, 16]. Actually, the purely phenomenological Skyrme force should be viewed as to provide a convenient parametrization of the nuclear energy density functional on which the self-consistent mean-field treatment can be based. Following the current efforts to build new functionals from many-body techniques, the second-order contributions arising from the Skyrme NN-contact interaction in nuclear matter have been calculated in ref.[17]. The second-order results obtained for various nuclear matter quantities consist of even powers of the Fermi momentum $k_{f}$ multiplied by products of the Skyrme parameters and a numerical coefficient of the form $\ln 2+r_{j}$, where $r_{j}$ is some rational number. This line of attempt has been continued recently by Moghrabi in ref.[18], where the nextto-next-to-leading order Skyrme interaction (quartic in momenta) has been considered and nuclear bulk quantities have been calculated from it up to second order. In view of these developments it is one particular aim of the present work to derive the (complete) third-order contributions from ring and ladder diagrams for the next-to-leading order Skyrme interaction (quadratic in momenta). The general NN-contact interaction of order $\mathcal{O}\left(p^{2}\right)$ is readily obtained by adding two tensor terms and their third-order contributions are evaluated together with all possible interference terms.

Let us remind that the third-order contributions known so far in the literature constitute two parts in the low-density expansion for an interacting many-fermion system [19], which read:

$$
\bar{E}\left(k_{f}\right)^{3-\text { ring }}=(1-g)(g-3) \frac{a^{3} k_{f}^{5}}{M \pi^{4}} \cdot 2.7950523, \quad \bar{E}\left(k_{f}\right)^{3-\mathrm{lad}}=(1-g) \frac{a^{3} k_{f}^{5}}{M \pi^{3}} \cdot 1.1716223 .
$$

These results for the energy per particle $\bar{E}\left(k_{f}\right)$ derive from a (momentum-independent) contactinteraction proportional to the s-wave scattering length $a$ (where $a>0$ corresponds to attraction) and $g$ is the spin-degeneracy factor entering the relation between the density $\rho=g k_{f}^{3} / 6 \pi^{2}$ and the Fermi momentum $k_{f}$. The large fermion mass is denoted by $M$. In the course of the present work it will also become clear, how the numerical coefficients can be computed with the given accuracy.

The present paper is organized as follows. In section 2 we prepare the evaluation of third-order particle-hole ring diagrams by introducing the antisymmetrized Skyrme NN-contact interaction which allows to treat in one single step direct and exchange-type contributions together. For the momentum-dependent interaction product resulting from the spin- and isospin-traces, three of the four loop-integrations can be factorized and solved in terms of cubic expressions in euclidean polarization functions. The results for the three-ring energy per particle of isospin-symmetric nuclear matter and pure neutron matter are given in section 3, where the focus lies on the computation of the pertinent four-loop coefficients with high numerical accuracy. Dimensional regularization of divergent integrals is performed by subtracting power-divergencs from the integrand. The validity of this practical method is checked against the known analytical results at second order. In section 4 the general $\mathcal{O}\left(p^{2}\right) \mathrm{NN}$-contact interactions is completed by introducing two additional tensor interactions and the corresponding third-order ring contributions are evaluated together with all possible interference terms. Section 5 is devoted to a semi-analytical evaluation of the third-order particle-hole ring diagrams with the long-range one-pion exchange interaction. It is found that exchange-type corrections are smaller than the direct three-ring contribution, but these add up coherently to a very large attraction of $\bar{E}\left(k_{f 0}\right) \simeq-92 \mathrm{MeV}$. Finally, in section 6 the contributions from the third-order (particle-particle and hole-hole) ladder diagrams are evaluated for the general $\mathcal{O}\left(p^{2}\right) \mathrm{NN}$-contact interaction. Moreover, in the appendix the three-ring contributions to the isospin-asymmetry energy $A\left(k_{f}\right) \sim k_{f}^{5}$ as they arise from a contact-interaction involving two scattering lengths $a_{s}, a_{t}$ are studied.

\section{Ring diagrams and antisymmetrized Skyrme-interaction}

The four topologically distinct ring diagrams representing the third-order particle-hole contribution to the energy density are shown in Fig. 1, organized according to the number of direct and exchange 

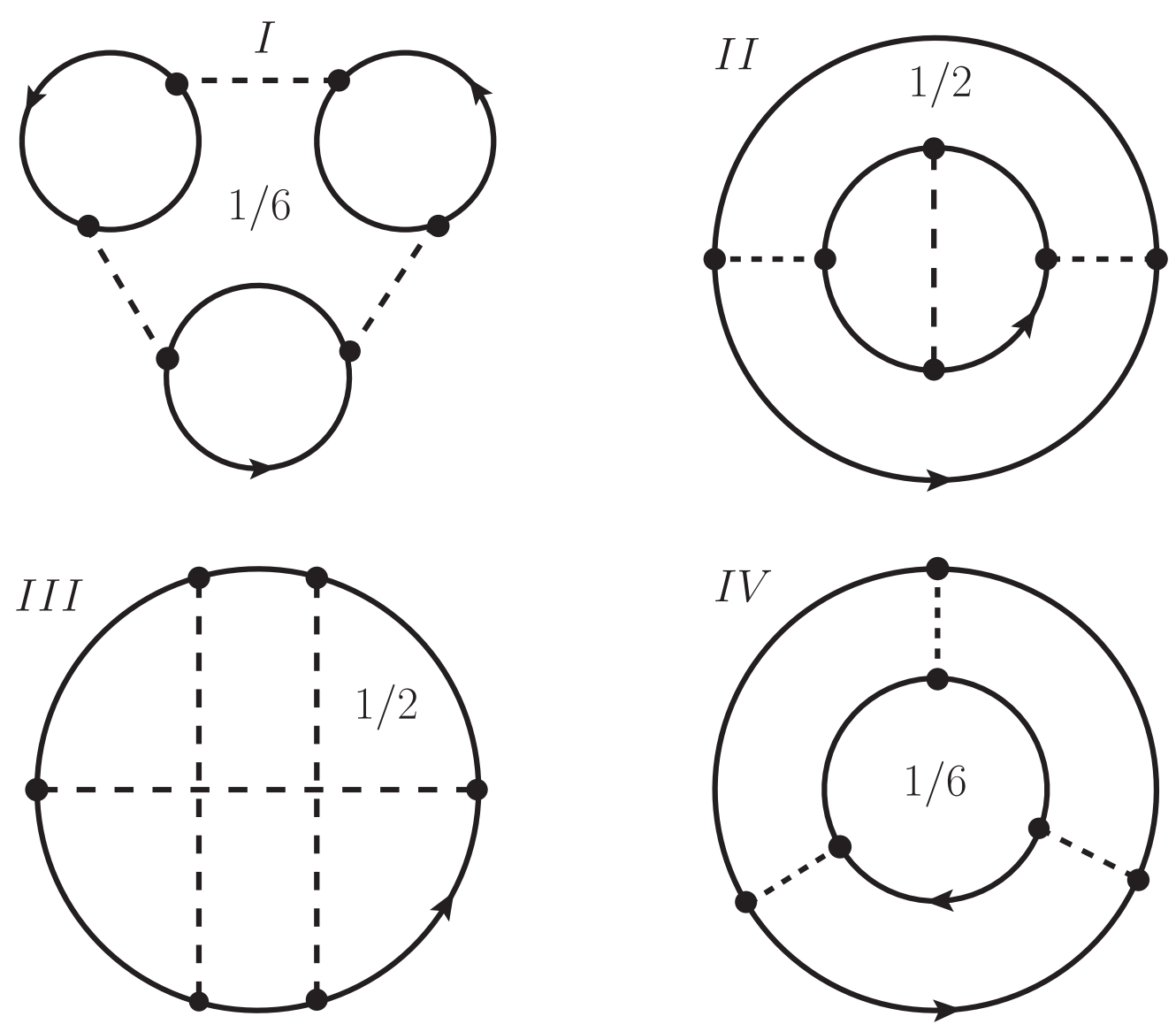

Figure 1: Third-order particle-hole ring diagrams: $I=\operatorname{dir}^{3}, I I=-\operatorname{dir}^{2}$ exc, $I I I=\operatorname{dir}^{2} \operatorname{exc}^{2}$ and $I V=-e x c^{3}$. The corresponding symmetry factors $1 / 6,1 / 2,1 / 2$ and $1 / 6$ are indicated. The dashed line represents the Skyrme contact-interaction $V_{\mathrm{Sk}}$ or one-pion exchange.

interactions. Together with their symmetry factors $1 / 6,1 / 2,1 / 2,1 / 6$ and signs from the number of closed fermion-lines these diagrams result formally from the expansion of $-1 / 6(\text { dir }-e x c)^{3}$. Therefore, the three exchange-type diagrams are automatically included by employing the antisymmetrized two-body interaction dir - exc. In the case of the Skyrme NN-contact interaction [17, the antisymmetrization operator leaves unchanged the momentum-dependence:

$$
\begin{array}{r}
V_{\mathrm{Sk}}-\left.P_{\sigma} P_{\tau} V_{\mathrm{Sk}}\right|_{\vec{q}_{\text {out } \rightarrow-\vec{q}_{\text {out }}}}=\left(1-P_{\sigma} P_{\tau}\right)\left\{t_{0}\left(1+x_{0} P_{\sigma}\right)+\frac{t_{1}}{2}\left(1+x_{1} P_{\sigma}\right)\left(\vec{q}_{\text {out }}^{2}+\vec{q}_{\text {in }}^{2}\right)\right\} \\
+\left(1+P_{\sigma} P_{\tau}\right) t_{2}\left(1+x_{2} P_{\sigma}\right) \vec{q}_{\text {out }} \cdot \vec{q}_{\text {in }}+\left(1+P_{\tau}\right) i W_{0}\left(\vec{\sigma}_{1}+\vec{\sigma}_{2}\right) \cdot\left(\vec{q}_{\text {out }} \times \vec{q}_{\text {in }}\right) .
\end{array}
$$

Here, $P_{\sigma}=\left(1+\vec{\sigma}_{1} \cdot \vec{\sigma}_{2}\right) / 2$ and $P_{\tau}=\left(1+\vec{\tau}_{1} \cdot \vec{\tau}_{2}\right) / 2$ are the spin- and isospin-exchange operators, while $\vec{q}_{\text {in }}=\left(\vec{p}_{1}-\vec{p}_{2}\right) / 2$ and $\vec{q}_{\text {out }}=\left(\vec{p}_{1}^{\prime}-\vec{p}_{2}^{\prime}\right) / 2$ denote half of the momentum difference in the initial and final state, respectively. Note that the anti-symmetrized spin-orbit contact-interaction $\sim W_{0}$ has been simplified by using the relation $\vec{\sigma}_{1} \cdot \vec{\sigma}_{2}\left(\vec{\sigma}_{1}+\vec{\sigma}_{2}\right)=\vec{\sigma}_{1}+\vec{\sigma}_{2}$. The three closed nucleon-lines of the third-order ring diagram $I$ in Fig. 1 are associated with triple spin- and isospin traces, which are readily computed with the help of the following master formula:

$$
\begin{aligned}
& \frac{1}{64} \operatorname{tr}_{1} \operatorname{tr}_{2} \operatorname{tr}_{3}\left\{\left(A+B \vec{\sigma}_{1} \cdot \vec{\sigma}_{2}+C \vec{\tau}_{1} \cdot \vec{\tau}_{2}+D \vec{\sigma}_{1} \cdot \vec{\sigma}_{2} \vec{\tau}_{1} \cdot \vec{\tau}_{2}\right)\left(A^{\prime}+B^{\prime} \vec{\sigma}_{2} \cdot \vec{\sigma}_{3}+C^{\prime} \vec{\tau}_{2} \cdot \vec{\tau}_{3}+D^{\prime} \vec{\sigma}_{2} \cdot \vec{\sigma}_{3} \vec{\tau}_{2} \cdot \vec{\tau}_{3}\right)\right. \\
& \left.\times\left(A^{\prime \prime}+B^{\prime \prime} \vec{\sigma}_{3} \cdot \vec{\sigma}_{1}+C^{\prime \prime} \vec{\tau}_{3} \cdot \vec{\tau}_{1}+D^{\prime \prime} \vec{\sigma}_{3} \cdot \vec{\sigma}_{1} \vec{\tau}_{3} \cdot \vec{\tau}_{1}\right)\right\}=A A^{\prime} A^{\prime \prime}+3 B B^{\prime} B^{\prime \prime}+3 C C^{\prime} C^{\prime \prime}+9 D D^{\prime} D^{\prime \prime} .(3)
\end{aligned}
$$

Applying the decomposition into $A, B, C, D$ to three antisymmetrized Skyrme NN-contact interactions and treating separately the spin-orbit term, one obtains the following expression for the 
interaction product:

$$
\begin{aligned}
& 12 t_{0}^{3}\left(1-6 x_{0}^{2}\right)+9 t_{0}^{2} t_{1}\left(1-2 x_{0}^{2}-4 x_{0} x_{1}\right)\left(\vec{l}_{12}^{2}+\vec{q}^{2}\right)+9 t_{0}^{2} t_{2}\left[5+4 x_{2}+2 x_{0}^{2}\left(1+2 x_{2}\right)\right]\left(\vec{l}_{12}^{2}-\vec{q}^{2}\right) \\
& +\frac{9}{4} t_{0} t_{1}^{2}\left(1-4 x_{0} x_{1}-2 x_{1}^{2}\right)\left(\vec{l}_{12}^{2} \vec{l}_{13}^{2}+2 \vec{l}_{12}^{2} \vec{q}^{2}+\vec{q}^{4}\right)+\frac{9}{2} t_{0} t_{1} t_{2}\left[5+4 x_{2}+2 x_{0} x_{1}\left(1+2 x_{2}\right)\right] \\
& \times\left(\vec{l}_{12}^{2} \vec{l}_{13}^{2}-\vec{q}^{4}\right)+\frac{9}{4} t_{0} t_{2}^{2}\left(5+8 x_{2}+2 x_{2}^{2}\right)\left(\vec{l}_{12}^{2} \vec{l}_{13}^{2}-2 \vec{l}_{12}^{2} \vec{q}^{2}+\vec{q}^{4}\right) \\
& +\frac{9}{16} t_{1}^{2} t_{2}\left[5+2 x_{1}^{2}+4 x_{2}\left(1+x_{1}^{2}\right)\right]\left(\vec{l}_{12}^{2} \vec{l}_{13}^{2} \vec{l}_{23}^{2}+\vec{l}_{12}^{2} \vec{l}_{13}^{2} \vec{q}^{2}-\vec{l}_{12}^{2} \vec{q}^{4}-\vec{q}^{6}\right) \\
& +\frac{9}{16} t_{1} t_{2}^{2}\left(5+8 x_{2}+2 x_{2}^{2}\right)\left(\vec{l}_{12}^{2} \vec{l}_{13}^{2} \vec{l}_{23}^{2}-\vec{l}_{12}^{2} \vec{l}_{13}^{2} \vec{q}^{2}-\vec{l}_{12}^{2} \vec{q}^{4}+\vec{q}^{6}\right) \\
& +\frac{3}{16} t_{1}^{3}\left(1-6 x_{1}^{2}\right)\left(\vec{l}_{12}^{2} \vec{l}_{13}^{2} \vec{l}_{23}^{2}+3 \vec{l}_{12}^{2} \vec{l}_{13}^{2} \vec{q}^{2}+3 \vec{l}_{12}^{2} \vec{q}^{4}+\vec{q}^{6}\right) \\
& +\frac{t_{2}^{3}}{16}\left(35+84 x_{2}+78 x_{2}^{2}+28 x_{2}^{3}\right)\left(\vec{l}_{12}^{2} \vec{l}_{13}^{2} \vec{l}_{23}^{2}-3 \vec{l}_{12}^{2} \vec{l}_{13}^{2} \vec{q}^{2}+3 \vec{l}_{12}^{2} \vec{q}^{4}-\vec{q}^{6}\right) \\
& +9 W_{0}^{2}\left(\vec{l}_{12} \times \vec{q}\right) \cdot\left(\vec{l}_{13} \times \vec{q}\right)\left\{4 t_{0}\left(1+x_{0}\right)+t_{1}\left(1+x_{1}\right)\left(\vec{l}_{23}^{2}+\vec{q}^{2}\right)+5 t_{2}\left(1+x_{2}\right)\left(\vec{l}_{23}^{2}-\vec{q}^{2}\right)\right\} .
\end{aligned}
$$

Here, $\vec{l}_{i j}=\vec{l}_{i}-\vec{l}_{j}$ denote differences of the loop-momenta $\vec{l}_{1}, \vec{l}_{2}, \vec{l}_{3}$ belonging to individual nucleon rings and $\vec{q}$ is the momentum running into and out of each ring (see first diagram in Fig. 1). Note that we have exploited the permutational symmetry in the indices (123) in order to reduce the number of independent terms. It is interesting to observe that, as a result of the triple spin-trace, the spin-orbit interaction $\sim W_{0}$ contributes only at quadratic order.

The next step in the evaluation of the energy density consists in performing four four-dimensional loop-integrations. The loop-integrations related to the three closed nucleon-rings can actually be factorized (by means of tensor contractions), because the interaction product in eq.(4) involves only products of scalar-products of the momenta $\vec{l}_{1}, \vec{l}_{2}, \vec{l}_{3}$ and $\vec{q}$. By exploiting this convenient factorization, the three internal (four-dimensional) loop-integrations can be solved analytically in terms of cubic expressions in a set of (euclidean) polarization functions 2

\subsection{Polarization functions}

An elementary way to derive the euclidean polarization function is to start from the finitetemperature formalism, with the baryon chemical potential set to $\mu=k_{f}^{2} / 2 M$, and to take the limit $T \rightarrow 0$ in the end. The sum over (internal) fermionic Matsubara frequencies leads to Fermi-Dirac distributions, which degenerate to step-functions in the limit $T \rightarrow 0$, while the external bosonic Matsubara frequency becomes a continuous variable $\omega$. Carrying out this procedure, the euclidean polarization function including a minus-sign from the closed fermion loop has the representation:

$$
\Pi(\omega, \vec{q})=\int \frac{d^{3} l}{(2 \pi)^{3}} \frac{1}{i \omega+\vec{l} \cdot \vec{q} / M}\left\{\theta\left(k_{f}-|\vec{l}-\vec{q} / 2|\right)-\theta\left(k_{f}-|\vec{l}+\vec{q} / 2|\right)\right\} .
$$

The integral in eq.(5) is most conveniently solved by substituting $\vec{l} \rightarrow-\vec{l}$ in the second term and then shifting $\vec{l} \rightarrow \vec{l}+\vec{q} / 2$. This way the difference of the two emerging energy denominators becomes real-valued. It is furthermore advantageous to introduce dimensionless variables $s$ and $\kappa$ by setting $|\vec{q}|=2 s k_{f}$ and $\omega=2 s \kappa k_{f}^{2} / M$. Altogether one finds:

$$
\Pi[1](\omega, \vec{q})=\frac{M k_{f}}{4 \pi^{2} s} Q_{0}(s, \kappa),
$$

\footnotetext{
${ }^{2}$ The advantage of the (basic) euclidean polarization function is that it is real-valued, whereas the one in Minkowski-space is complex-valued (see ref. [20]).
} 
with the dimensionless function:

$$
Q_{0}(s, \kappa)=s-s \kappa \arctan \frac{1+s}{\kappa}-s \kappa \arctan \frac{1-s}{\kappa}+\frac{1}{4}\left(1-s^{2}+\kappa^{2}\right) \ln \frac{(1+s)^{2}+\kappa^{2}}{(1-s)^{2}+\kappa^{2}} .
$$

It is important to note that this expression for $Q_{0}(s, \kappa)$ agrees perfectly with ref.[21], where a completely different method has been used to derive the polarization function. For the purpose of factorizing products of scalar-products (of $\vec{l}_{1}, \vec{l}_{2}, \vec{l}_{3}$ and $\vec{q}$ ) one needs additional polarization functions, which involve one or two $\vec{l}$-factors:

$$
\Pi[\vec{l}]=-\frac{M k_{f}^{2}}{4 \pi^{2} s} i \kappa Q_{0}(s, \kappa) \hat{q}, \quad \Pi\left[l_{i} l_{j}\right]=\frac{M k_{f}^{3}}{4 \pi^{2} s}\left\{\frac{\delta_{i j}}{3} Q_{1}(s, \kappa)+\left(\hat{q}_{i} \hat{q}_{j}-\frac{\delta_{i j}}{3}\right) Q_{2}(s, \kappa)\right\}
$$

where

$$
\begin{gathered}
Q_{1}(s, \kappa)=\left(1-s^{2}\right) Q_{0}(s, \kappa)+\frac{s}{2}\left(1+s^{2}+\kappa^{2}\right)-\frac{1}{8}\left[(1+s)^{2}+\kappa^{2}\right]\left[(1-s)^{2}+\kappa^{2}\right] \ln \frac{(1+s)^{2}+\kappa^{2}}{(1-s)^{2}+\kappa^{2}}, \\
Q_{2}(s, \kappa)=s-\frac{1}{2} Q_{1}(s, \kappa)-\frac{3 \kappa^{2}}{2} Q_{0}(s, \kappa),
\end{gathered}
$$

as well as those involving three or four $\vec{l}$-factors combined to a vector or a scalar:

$$
\Pi\left[\vec{l}^{2} \vec{l}\right]=-\frac{M k_{f}^{4}}{4 \pi^{2} s} i \kappa Q_{1}(s, \kappa) \hat{q}, \quad \Pi\left[\vec{l}^{4}\right]=\frac{M k_{f}^{5}}{4 \pi^{2} s} Q_{3}(s, \kappa)
$$

where

$$
Q_{3}(s, \kappa)=\frac{16 s^{3}}{9}+\frac{2}{3}\left(2-2 s^{2}-\kappa^{2}\right) Q_{1}(s, \kappa)+\frac{1}{3}\left[2 \kappa^{2}\left(1-3 s^{2}\right)-\left(s^{2}-1\right)^{2}\right] Q_{0}(s, \kappa) .
$$

It is worth to note that all dimensionless polarization functions $Q_{j}(s, \kappa)$ are even under $\kappa \rightarrow-\kappa$.

\section{$3 \quad$ Energy per particle}

In the previous section we have assembled all the necessary ingredients to evaluate the particle-hole ring diagrams at four-loop order. The remaining four-dimensional loop-integral $(2 \pi)^{-4} \int_{-\infty}^{\infty} d \omega \int d^{3} q$ can be reduced to a double-integral of the form $\left(8 k_{f}^{5} / M \pi^{3}\right) \int_{0}^{\infty} d \kappa \int_{0}^{\infty} d s s^{3}$. By adapting the pattern of cubic terms in the Skyrme parameters $t_{j}, x_{j}, W_{0}$ as specified by the interaction product in eq. (4), one arrives at the following expression for the three-ring energy per particle of isospin-symmetric nuclear matter (with density $\rho=2 k_{f}^{3} / 3 \pi^{2}$ ):

$$
\begin{aligned}
\bar{E}\left(k_{f}\right)^{(3-\text { ring })=}= & \frac{M^{2} k_{f}^{5}}{32 \pi^{7}}\left\{t_{0}^{3}\left(1-6 x_{0}^{2}\right) \mathcal{N}_{1}+k_{f}^{2} t_{0}^{2} t_{1}\left(1-2 x_{0}^{2}-4 x_{0} x_{1}\right) \mathcal{N}_{2}\right. \\
& +k_{f}^{2} t_{0}^{2} t_{2}\left[5+4 x_{2}+2 x_{0}^{2}\left(1+2 x_{2}\right)\right] \mathcal{N}_{3}+k_{f}^{4} t_{0} t_{1}^{2}\left(4 x_{0} x_{1}+2 x_{1}^{2}-1\right) \mathcal{N}_{4} \\
& +k_{f}^{4} t_{0} t_{1} t_{2}\left[\frac{5}{2}+x_{0} x_{1}\left(1+2 x_{2}\right)+2 x_{2}\right] \mathcal{N}_{5}+k_{f}^{4} t_{0} t_{2}^{2}\left[\frac{5}{2}+4 x_{2}+x_{2}^{2}\right] \mathcal{N}_{6} \\
& +k_{f}^{6} t_{1}^{2} t_{2}\left[\frac{5}{2}+x_{1}^{2}+2 x_{2}\left(1+x_{1}^{2}\right)\right] \mathcal{N}_{7}+k_{f}^{6} t_{1} t_{2}^{2}\left[\frac{5}{2}+4 x_{2}+x_{2}^{2}\right] \mathcal{N}_{8} \\
& +k_{f}^{6} t_{1}^{3}\left(1-6 x_{1}^{2}\right) \mathcal{N}_{9}+k_{f}^{6} t_{2}^{3}\left[\frac{5}{4}+3 x_{2}+\frac{39}{14} x_{2}^{2}+x_{2}^{3}\right] \mathcal{N}_{10} \\
& \left.+k_{f}^{4} W_{0}^{2}\left[t_{0}\left(1+x_{0}\right) \mathcal{N}_{11}+k_{f}^{2} t_{1}\left(1+x_{1}\right) \mathcal{N}_{12}+k_{f}^{2} t_{2}\left(1+x_{2}\right) \mathcal{N}_{13}\right]\right\} .
\end{aligned}
$$


The proportionality of $\bar{E}\left(k_{f}\right)^{(3-\text { ring })}$ to the squared nucleon mass $M^{2}$ is obvious for a third-order contribution and the occurring odd powers of the Fermi momentum $k_{f}$ are predetermined by the dimensions of the coupling parameters: $t_{0} \sim \mathrm{fm}^{2}$ and $t_{1,2}, W_{0} \sim \mathrm{fm}^{4}$. Beyond these scaling properties, the entire many-body dynamics as represented by the third-order particle-hole ring diagrams is encoded in the numerical coefficients $\mathcal{N}_{j}, j=1, \ldots, 13$, which will be computed in the next subsection. For comparison, we consider also the third-order particle-hole contribution in pure neutron matter. In this case the isospin-exchange operator $P_{\tau} \rightarrow 1$ and the antisymmetrized Skyrme $n n$-contact interaction depends only on the parameter combinations $t_{0}\left(1-x_{0}\right), t_{1}(1-$ $\left.x_{1}\right), t_{2}\left(1+x_{2}\right)$ and $W_{0}$. Taking care of these modifications, one finds for the three-ring energy per particle in pure neutron matter (with density $\rho_{n}=k_{n}^{3} / 3 \pi^{2}$ ):

$$
\begin{aligned}
\bar{E}_{n}\left(k_{n}\right)^{(3-\text { ring })=} & \frac{M^{2} k_{n}^{5}}{96 \pi^{7}}\left\{t_{0}^{3}\left(x_{0}-1\right)^{3} \mathcal{N}_{1}+k_{n}^{2} t_{0}^{2} t_{1}\left(x_{0}-1\right)^{2}\left(x_{1}-1\right) \mathcal{N}_{2}\right. \\
& +k_{n}^{2} t_{0}^{2} t_{2}\left(x_{0}-1\right)^{2}\left(x_{2}+1\right) 3 \mathcal{N}_{3}+k_{n}^{4} t_{0} t_{1}^{2}\left(1-x_{0}\right)\left(x_{1}-1\right)^{2} \mathcal{N}_{4} \\
& +k_{n}^{4} t_{0} t_{1} t_{2}\left(x_{0}-1\right)\left(x_{1}-1\right)\left(x_{2}+1\right) \frac{3 \mathcal{N}_{5}}{2}+k_{n}^{4} t_{0} t_{2}^{2}\left(1-x_{0}\right)\left(1+x_{2}\right)^{2} \frac{3 \mathcal{N}_{6}}{2} \\
& +k_{n}^{6} t_{1}^{2} t_{2}\left(1-x_{1}\right)^{2}\left(1+x_{2}\right) \frac{3 \mathcal{N}_{7}}{2}+k_{n}^{6} t_{1} t_{2}^{2}\left(1-x_{1}\right)\left(1+x_{2}\right)^{2} \frac{3 \mathcal{N}_{8}}{2} \\
& \left.+k_{n}^{6} t_{1}^{3}\left(x_{1}-1\right)^{3} \mathcal{N}_{9}+k_{n}^{6} t_{2}^{3}\left(1+x_{2}\right)^{3} \frac{45 \mathcal{N}_{10}}{28}+k_{n}^{6} W_{0}^{2} t_{2}\left(1+x_{2}\right) \frac{8 \mathcal{N}_{13}}{5}\right\},
\end{aligned}
$$

which differs from eq.(13) by certain reduction factors at the coefficients $\mathcal{N}_{j}$. Evidently, the terms proportional to $W_{0}^{2} t_{0}\left(1+x_{0}\right) \mathcal{N}_{11}$ and $W_{0}^{2} t_{1}\left(1+x_{1}\right) \mathcal{N}_{12}$ have dropped out in $\bar{E}_{n}\left(k_{n}\right)^{(3-\text { ring })}$ since these involve a coupling to the Pauli-forbidden ${ }^{3} S_{1}$-state of two neutrons.

\subsection{Calculation of four-loop coefficients}

We are left with the task to compute numerically the four-loop coefficients $\mathcal{N}_{j}$. In the case of the pure s-wave contact-interaction $t_{0}\left(1+x_{0} P_{\sigma}\right)$ this concerns $\mathcal{N}_{1}$, which is calculated most efficiently by introducing polar coordinates, $s=r \cos \varphi, \kappa=r \sin \varphi$, in the $s \kappa$ quarter-plane:

$$
\mathcal{N}_{1}=12 \int_{0}^{\infty} d r \int_{0}^{\pi / 2} d \varphi r\left[Q_{0}(s, \kappa)\right]^{3}=4.1925784
$$

At this point one can remark that the coefficient in the left part of eq.(1) is $2 \mathcal{N}_{1} / 3=2.7950523$. The s-wave effective range correction $\sim t_{1}\left(1+x_{1} P_{\sigma}\right)$ is involved linearly in the second terms in eqs. $(13,14)$ and the corresponding coefficient $\mathcal{N}_{2}$ is determined by an integral over $Q_{0}^{2}\left[Q_{1}+\left(2 s^{2}+\kappa^{2}\right) Q_{0}\right]$, which however diverges. We apply dimensional regularization in a practical and empirical way by subtracting those terms from the integrand which lead to power divergencies $r_{\max }^{2 n+1}$ in the radial integration $\int_{0}^{r_{\max }} d r$ up to a cutoff $r_{\max }$. After implementing this regularization the (convergent) coefficient $\mathcal{N}_{2}$ is given by:

$$
\mathcal{N}_{2}=\int_{0}^{\infty} d r \int_{0}^{\pi / 2} d \varphi\left\{18 r Q_{0}^{2}\left[Q_{1}+\left(2 s^{2}+\kappa^{2}\right) Q_{0}\right]-\frac{16}{3} \cos ^{3} \varphi(2+\cos 2 \varphi)\right\}=-0.4633512
$$

In the actual calculation it is most advantageous to expand the integrand further in powers of $r^{-2}$ up to order $r^{-4}$. The value $\int_{0}^{\pi / 2} d \varphi\left\{f_{2}(\cos \varphi) / r_{\max }+f_{4}(\cos \varphi) / 3 r_{\max }^{3}\right\}$ is then included as a good approximation of the contribution to the double-integral from the outside region $r>r_{\max }$. The angular functions $f_{2,4}(\cos \varphi)$ emerging from the $1 / r^{2}$-expansion are always odd polynomials in 
$\cos \varphi$. By applying this procedure one gets numerically accurate and well-converged results from the restricted radial integral $\int_{0}^{r_{\max }} d r$ with $r_{\max }$ in the range 20 to 50. The regularized coefficient $\mathcal{N}_{3}$ for the ssp-wave interference term proportional to $t_{0}^{2} t_{2}$ in eqs. $(13,14)$ reads:

$$
\mathcal{N}_{3}=\int_{0}^{\infty} d r \int_{0}^{\pi / 2} d \varphi\left\{18 r Q_{0}^{2}\left[Q_{1}+\left(\kappa^{2}-2 s^{2}\right) Q_{0}\right]+\frac{16}{3} \cos ^{3} \varphi \cos 2 \varphi\right\}=-2.259163
$$

The product $\vec{l}_{12}^{2} \vec{l}_{13}^{2}$ of two squared momentum-differences leads to the combination of polarization functions:

$$
\mathbf{C}_{a}=3 Q_{0} Q_{1}^{2}+Q_{0}^{2}\left[Q_{3}+\frac{4 \kappa^{2}}{3}\left(5 Q_{1}-2 Q_{2}\right)\right]
$$

which allows us to compute the next three coefficients:

$$
\begin{gathered}
\mathcal{N}_{4}=-9 \int_{0}^{\infty} d r \int_{0}^{\pi / 2} d \varphi\left\{\frac{r}{4} \mathbf{C}_{a}+4 r s^{2} Q_{0}^{2}\left[Q_{1}+\left(s^{2}+\kappa^{2}\right) Q_{0}\right]\right\}_{\mathrm{reg}}=2.902123 \\
\mathcal{N}_{5}=9 \int_{0}^{\infty} d r \int_{0}^{\pi / 2} d \varphi\left\{r\left(\mathbf{C}_{a}-16 s^{4} Q_{0}^{3}\right)\right\}_{\mathrm{reg}}=2.126584 \\
\mathcal{N}_{6}=9 \int_{0}^{\infty} d r \int_{0}^{\pi / 2} d \varphi\left\{\frac{r}{2} \mathbf{C}_{a}+8 r s^{2} Q_{0}^{2}\left[\left(s^{2}-\kappa^{2}\right) Q_{0}-Q_{1}\right]\right\}_{\mathrm{reg}}=0.438970
\end{gathered}
$$

where the subscript 'reg' indicates the subtraction of power divergences proportional to $r^{2 n}, n=$ $0,1,2$. The product $\vec{l}_{12}^{2} \vec{l}_{13}^{2} \vec{l}_{23}^{2}$ of three squared momentum-differences leads to twice the combination:

$$
\mathbf{C}_{b}=\kappa^{2} Q_{0}\left(5 Q_{1}^{2}-8 Q_{1} Q_{2}+3 Q_{0} Q_{3}\right)+3 Q_{0} Q_{1} Q_{3}+\frac{5}{9} Q_{1}^{3}-\frac{8}{9} Q_{2}^{2}\left(3 Q_{1}+Q_{2}\right),
$$

which appears together with $\mathbf{C}_{a}$ in the evaluation of the next four coefficients:

$$
\begin{gathered}
\mathcal{N}_{7}=9 \int_{0}^{\infty} d r \int_{0}^{\pi / 2} d \varphi\left\{\frac{r}{4}\left(\mathbf{C}_{b}+2 s^{2} \mathbf{C}_{a}\right)-4 r s^{4} Q_{0}^{2}\left[Q_{1}+\left(2 s^{2}+\kappa^{2}\right) Q_{0}\right]\right\}_{\text {reg }}=0.48756 \\
\mathcal{N}_{8}=9 \int_{0}^{\infty} d r \int_{0}^{\pi / 2} d \varphi\left\{\frac{r}{4}\left(\mathbf{C}_{b}-2 s^{2} \mathbf{C}_{a}\right)+4 r s^{4} Q_{0}^{2}\left[\left(2 s^{2}-\kappa^{2}\right) Q_{0}-Q_{1}\right]\right\}_{\text {reg }}=-0.27614 \\
\mathcal{N}_{9}=3 \int_{0}^{\infty} d r \int_{0}^{\pi / 2} d \varphi\left\{\frac{r}{8}\left(\mathbf{C}_{b}+6 s^{2} \mathbf{C}_{a}\right)+2 r s^{4} Q_{0}^{2}\left[3 Q_{1}+\left(2 s^{2}+3 \kappa^{2}\right) Q_{0}\right]\right\}_{\text {reg }}=-1.01924 \\
\mathcal{N}_{10}=7 \int_{0}^{\infty} d r \int_{0}^{\pi / 2} d \varphi\left\{\frac{r}{2}\left(\mathbf{C}_{b}-6 s^{2} \mathbf{C}_{a}\right)+8 r s^{4} Q_{0}^{2}\left[3 Q_{1}+\left(3 \kappa^{2}-2 s^{2}\right) Q_{0}\right]\right\}_{\text {reg }}=0.315484
\end{gathered}
$$


The coefficients belonging to the last three terms in eq.(13) involving the spin-orbit coupling $W_{0}$ at quadratic order, read finally:

$$
\begin{gathered}
\mathcal{N}_{11}=16 \int_{0}^{\infty} d r \int_{0}^{\pi / 2} d \varphi\left\{6 r s^{2} Q_{0}^{2}\left(Q_{1}-Q_{2}\right)-\frac{16}{15} \cos ^{5} \varphi\right\}=-2.244200 \\
\mathcal{N}_{12}=16 \int_{0}^{\infty} d r \int_{0}^{\pi / 2} d \varphi\left\{r s^{2} Q_{0}\left(Q_{1}-Q_{2}\right)\left[2 Q_{1}+Q_{2}+3\left(2 s^{2}+\kappa^{2}\right) Q_{0}\right]\right\}_{\text {reg }}=-2.30577 \\
\mathcal{N}_{13}=80 \int_{0}^{\infty} d r \int_{0}^{\pi / 2} d \varphi\left\{r s^{2} Q_{0}\left(Q_{1}-Q_{2}\right)\left[2 Q_{1}+Q_{2}+3\left(\kappa^{2}-2 s^{2}\right) Q_{0}\right]\right\}_{\text {reg }}=2.53887
\end{gathered}
$$

\subsection{Second order calculation}

In the previous subsection dimensional regularization of the divergent integrals $\mathcal{N}_{j}$ has been performed by subtracting power divergences. We will now verify this empirical method by rederiving the known analytical results for the Skyrme NN-contact interaction at second order [17]. The many-body contributions at second order are usually categorized into the particle-particle ladder series. But the particle-particle ladder diagrams at second order (see Fig. 1 in ref.[17]) can be equally well interpreted as the second-order two-ring diagrams of the particle-hole type. When using the antisymmetrized NN-interaction one has to include (in addition to symmetry factor $1 / 4$ ) an extra factor $1 / 2$ in order not to double-count via $(\operatorname{dir}-e x c)^{2}=d i r^{2}+e x c^{2}-2 \operatorname{dir}$ exc the direct (Hartree) and exchange (Fock) term. Performing the calculation of the two-ring diagrams with the Skyrme NN-contact interaction, one obtains for the energy per particle at second order:

$$
\begin{aligned}
\bar{E}\left(k_{f}\right)^{(2 \mathrm{nd})}= & \frac{3 M k_{f}^{4}}{32 \pi^{5}}\left\{t_{0}^{2}\left(1+x_{0}^{2}\right) \mathcal{Z}_{1}+k_{f}^{2} t_{0} t_{1}\left(1+x_{0} x_{1}\right) \mathcal{Z}_{2}\right. \\
& \left.+k_{f}^{4} t_{1}^{2}\left(1+x_{1}^{2}\right) \mathcal{Z}_{3}+k_{f}^{4} t_{2}^{2}\left(5+8 x_{2}+5 x_{2}^{2}\right) \mathcal{Z}_{4}+k_{f}^{4} W_{0}^{2} \mathcal{Z}_{5}\right\} \\
\bar{E}_{n}\left(k_{n}\right)^{(2 \mathrm{nd})}= & \frac{M k_{n}^{4}}{32 \pi^{5}}\left\{t_{0}^{2}\left(1-x_{0}\right)^{2} \mathcal{Z}_{1}+k_{n}^{2} t_{0} t_{1}\left(1-x_{0}\right)\left(1-x_{1}\right) \mathcal{Z}_{2}\right. \\
& \left.+k_{n}^{4} t_{1}^{2}\left(1-x_{1}\right)^{2} \mathcal{Z}_{3}+k_{n}^{4} t_{2}^{2}\left(1+x_{2}\right)^{2} 9 \mathcal{Z}_{4}+k_{n}^{4} W_{0}^{2} 2 \mathcal{Z}_{5}\right\}
\end{aligned}
$$

where the coefficients $\mathcal{Z}_{j}$ are given by double-integrals $\int_{0}^{\infty} d \kappa \int_{0}^{\infty} d s s$ over quadratic expressions in the euclidean polarization functions $Q_{j}(s, \kappa)$. After implementing dimensional regularization through the subtraction of power divergences, these five coefficients read:

$$
\begin{gathered}
\mathcal{Z}_{1}=-8 \int_{0}^{\infty} d r \int_{0}^{\pi / 2} d \varphi\left\{3 r s Q_{0}^{2}-\frac{4}{3} \cos ^{3} \varphi\right\}=3.451697=\frac{4 \pi}{35}(11-2 \ln 2) \\
\mathcal{Z}_{2}=-24 \int_{0}^{\infty} d r \int_{0}^{\pi / 2} d \varphi\left\{r s Q_{0}\left[Q_{1}+\left(2 s^{2}+\kappa^{2}\right) Q_{0}\right]\right\}_{\text {reg }}=3.99902=\frac{8 \pi}{945}(167-24 \ln 2), \\
\mathcal{Z}_{3}=-\int_{0}^{\infty} d r \int_{0}^{\pi / 2} d \varphi\left\{r s\left[24 s^{2}\left(s^{2}+\kappa^{2}\right) Q_{0}^{2}+3 Q_{0}\left(4\left(2 s^{2}+\kappa^{2}\right) Q_{1}+Q_{3}\right)+5 Q_{1}^{2}+4 Q_{2}^{2}\right]\right\}_{\text {reg }} \\
=1.37573=\frac{\pi}{10395}(4943-564 \ln 2),
\end{gathered}
$$




$$
\begin{aligned}
\mathcal{Z}_{4} & =-\frac{1}{3} \int_{0}^{\infty} d r \int_{0}^{\pi / 2} d \varphi\left\{r s\left[24 s^{2}\left(s^{2}-\kappa^{2}\right) Q_{0}^{2}+3 Q_{0}\left(4\left(\kappa^{2}-2 s^{2}\right) Q_{1}+Q_{3}\right)+5 Q_{1}^{2}+4 Q_{2}^{2}\right]\right\}_{\text {reg }} \\
& =0.0931718=\frac{\pi}{31185}(1033-156 \ln 2) \\
\mathcal{Z}_{5} & =128 \int_{0}^{\infty} d r \int_{0}^{\pi / 2} d \varphi\left\{r s^{3} Q_{0}\left(Q_{2}-Q_{1}\right)\right\}_{\text {reg }}=2.70935=\frac{16 \pi}{10395}(631-102 \ln 2)
\end{aligned}
$$

In each case the last entry gives the analytical value of $\mathcal{Z}_{j}$ as derived in section 3 of refs. [17] and one finds perfect agreement with the numerical evaluation of the regularized double-integrals. This serves as an important check on our approach which utilizes the subtraction of power divergences after the special choice of polar coordinates $s=r \cos \varphi, \kappa=r \sin \varphi$.

\section{Tensor interactions}

The Skyrme NN-contact interaction in eq.(2) depends on seven parameters. These are two less than entering the general $\mathcal{O}\left(p^{2}\right) \mathrm{NN}$-contact potential of chiral effective field theory [1, 2, 3]. A complete matching is achieved by adding to $V_{\mathrm{Sk}}$ the sum of two Galilei-invariant tensor interactions, whose antisymmetrized form reads:

$$
\begin{gathered}
V_{\text {ten }}-\left.P_{\sigma} P_{\tau} V_{\text {ten }}\right|_{\vec{q}_{\text {out }} \rightarrow-\vec{q}_{\text {out }}}=\left(1-P_{\tau}\right) t_{4}\left\{\vec{\sigma}_{1} \cdot \vec{q}_{\text {out }} \vec{\sigma}_{2} \cdot \vec{q}_{\text {out }}+\vec{\sigma}_{1} \cdot \vec{q}_{\text {in }} \vec{\sigma}_{2} \cdot \vec{q}_{\text {in }}-\frac{1}{3} \vec{\sigma}_{1} \cdot \vec{\sigma}_{2}\left(\vec{q}_{\text {out }}^{2}+\vec{q}_{\text {in }}^{2}\right)\right\} \\
+\left(1+P_{\tau}\right) t_{5}\left\{\vec{\sigma}_{1} \cdot \vec{q}_{\text {out }} \vec{\sigma}_{2} \cdot \vec{q}_{\text {in }}+\vec{\sigma}_{1} \cdot \vec{q}_{\text {in }} \vec{\sigma}_{2} \cdot \vec{q}_{\text {out }}-\frac{2}{3} \vec{\sigma}_{1} \cdot \vec{\sigma}_{2} \vec{q}_{\text {out }} \cdot \vec{q}_{\text {in }}\right\} .
\end{gathered}
$$

Note that the spin-exchange operator $P_{\sigma}$ does not modify the two tensor expressions in the curly brackets: $P_{\sigma}\{\ldots\}=\{\ldots\}$. It is also obvious that the $t_{4}$-term in eq.(37) vanishes in pure neutron matter. The second order contributions from $V_{\text {ten }}$, when calculated alternatively from the two-ring particle-hole diagrams, yield the following expressions for the energy per particle:

$$
\bar{E}\left(k_{f}\right)^{(2 \mathrm{nd})}=\frac{3 M k_{f}^{8}}{32 \pi^{5}}\left\{t_{4}^{2} \mathcal{Z}_{6}+t_{5}^{2} \mathcal{Z}_{7}\right\}, \quad \bar{E}_{n}\left(k_{n}\right)^{(2 \mathrm{nd})}=\frac{M k_{n}^{8}}{16 \pi^{5}} t_{5}^{2} \mathcal{Z}_{7}
$$

with the coefficients $\mathcal{Z}_{6}$ and $\mathcal{Z}_{7}$ given by:

$$
\begin{aligned}
\mathcal{Z}_{6} & =-\frac{16}{9} \int_{0}^{\infty} d r \int_{0}^{\pi / 2} d \varphi\left\{r s\left[24 s^{2}\left(s^{2}+\kappa^{2}\right) Q_{0}^{2}+3 Q_{0}\left(4 \kappa^{2} Q_{1}+8 s^{2} Q_{2}+Q_{3}\right)+5 Q_{1}^{2}+4 Q_{2}^{2}\right]\right\}_{\text {reg }} \\
& =1.54263=\frac{32 \pi}{93555}(1525-129 \ln 2), \\
\mathcal{Z}_{7} & =-\frac{16}{3} \int_{0}^{\infty} d r \int_{0}^{\pi / 2} d \varphi\left\{r s\left[24 s^{2}\left(s^{2}-\kappa^{2}\right) Q_{0}^{2}+3 Q_{0}\left(4 \kappa^{2} Q_{1}-8 s^{2} Q_{2}+Q_{3}\right)+5 Q_{1}^{2}+4 Q_{2}^{2}\right]\right\}_{\text {reg }} \\
& =4.200099=\frac{32 \pi}{405}(19-3 \ln 2) .
\end{aligned}
$$

In both cases one finds perfect agreement between the analytical value derived in section 5 of ref.[17] and the numerical evaluation of a regularized double-integral. 
Our next task consists in evaluating the third-order particle-hole ring diagrams with the tensorial contact-interaction in eq.(37) taking into account also all possible interference terms with the Skyrme NN-contact interaction. After some tedious algebra related to triple spin-traces one finds the following contribution to the three-ring energy per particle of isospin-symmetric nuclear matter:

$$
\begin{aligned}
\bar{E}\left(k_{f}\right)^{(3-\text { ring })=}= & \frac{M^{2} k_{f}^{9}}{32 \pi^{7}}\left\{k_{f}^{2} W_{0}^{2}\left[t_{4} \mathcal{N}_{14}+t_{5} \mathcal{N}_{15}\right]\right. \\
& +t_{4}^{2}\left[t_{0}\left(x_{0}-2\right) \mathcal{N}_{16}+k_{f}^{2} t_{1}\left(x_{1}-2\right) \mathcal{N}_{17}+k_{f}^{2} t_{2}\left(x_{2}+2\right) \mathcal{N}_{18}\right] \\
& +t_{4} t_{5}\left[t_{0} x_{0} \mathcal{N}_{19}+k_{f}^{2} t_{1} x_{1} \mathcal{N}_{20}+k_{f}^{2} t_{2} x_{2} \mathcal{N}_{21}\right] \\
& +t_{5}^{2}\left[t_{0}\left(3 x_{0}-2\right) \mathcal{N}_{22}+k_{f}^{2} t_{1}\left(3 x_{1}-2\right) \mathcal{N}_{23}+k_{f}^{2} t_{2}\left(3 x_{2}+2\right) \mathcal{N}_{24}\right] \\
& \left.+k_{f}^{2}\left[t_{4}^{3} \mathcal{N}_{25}+t_{4}^{2} t_{5} \mathcal{N}_{26}+t_{4} t_{5}^{2} \mathcal{N}_{27}+t_{5}^{3} \mathcal{N}_{28}\right]\right\}
\end{aligned}
$$

For pure neutron matter the analogous result is considerably simpler:

$$
\begin{aligned}
\bar{E}_{n}\left(k_{n}\right)^{(3-\text { ring })=} & \frac{M^{2} k_{n}^{9}}{24 \pi^{7}}\left\{k_{n}^{2} \frac{2 t_{5}}{5}\left[W_{0}^{2} \mathcal{N}_{15}+t_{5}^{2} \mathcal{N}_{28}\right]\right. \\
& \left.+t_{5}^{2}\left[t_{0}\left(x_{0}-1\right) \mathcal{N}_{22}+k_{n}^{2} t_{1}\left(x_{1}-1\right) \mathcal{N}_{23}+k_{n}^{2} t_{2}\left(x_{2}+1\right) \mathcal{N}_{24}\right]\right\},
\end{aligned}
$$

because the tensor term $\sim t_{4}$ responsible for ${ }^{3} S_{1}-{ }^{3} D_{1}$ mixing is not operative. We start with the coefficients of the terms in eqs. $(41,42)$ involving the squared spin-orbit parameter $W_{0}^{2}$, which read:

$$
\begin{aligned}
& \mathcal{N}_{14}=\frac{16}{3} \int_{0}^{\infty} d r \int_{0}^{\pi / 2} d \varphi\left\{r s^{2} Q_{0}\left(Q_{1}-Q_{2}\right)\left[5 Q_{1}-11 Q_{2}-6\left(2 s^{2}+\kappa^{2}\right) Q_{0}\right]\right\}_{\mathrm{reg}}=0.872202 \\
& \mathcal{N}_{15}=\frac{80}{3} \int_{0}^{\infty} d r \int_{0}^{\pi / 2} d \varphi\left\{r s^{2} Q_{0}\left(Q_{1}-Q_{2}\right)\left[5 Q_{1}-11 Q_{2}+6\left(2 s^{2}-\kappa^{2}\right) Q_{0}\right]\right\}_{\text {reg }}=-5.01745 .
\end{aligned}
$$

In the process of factorizing the occurring products of scalar-products 3 (of $\vec{l}_{1}, \vec{l}_{2}, \vec{l}_{3}$ and $\vec{q}$ ) one encounters new polarization functions:

$$
\Pi\left[\vec{l}^{2}(\vec{l} \cdot \hat{q})^{2}\right]=\frac{M k_{f}^{5}}{4 \pi^{2} s} Q_{4}(s, \kappa), \quad \Pi\left[(\vec{l} \cdot \hat{q})^{3}\right]=-\frac{M k_{f}^{4}}{4 \pi^{2} s} i \kappa Q_{5}(s, \kappa),
$$

which introduce the dimensionless functions:

$$
Q_{4}(s, \kappa)=\frac{2 s}{3}\left(1+s^{2}\right)-\kappa^{2} Q_{1}(s, \kappa), \quad Q_{5}(s, \kappa)=\frac{2 s}{3}-\kappa^{2} Q_{0}(s, \kappa),
$$

where $Q_{1}(s, \kappa)$ is written in eq. (9) and $Q_{0}(s, \kappa)$ in eq.(7). The interference terms quadratic in $t_{4}, t_{5}$ lead to the following three combinations of the dimensionless polarization functions:

$$
\begin{gathered}
\mathbf{K}_{a}=2 Q_{0}^{2}\left[Q_{3}+\frac{2 \kappa^{2}}{3}\left(Q_{1}+5 Q_{2}\right)\right]+6 Q_{0} Q_{2}^{2} \\
\mathbf{K}_{b}=8 s^{2}\left\{Q_{0}^{2}\left[3 Q_{4}-Q_{3}+\frac{2 \kappa^{2}}{3}\left(5 Q_{1}-2 Q_{2}+9 Q_{5}\right)\right]+6 Q_{0} Q_{1} Q_{2}\right\}
\end{gathered}
$$

\footnotetext{
${ }^{3}$ For example: $\left(\vec{l}_{1} \cdot \vec{l}_{2}\right)^{3}$ leads to the cubic expression $\kappa^{2} Q_{0}\left(6 Q_{1} Q_{5}-3 Q_{1}^{2}-5 Q_{5}^{2}\right) / 2$, and $\left(\overrightarrow{l_{1}} \cdot \overrightarrow{l_{2}}\right)\left(\overrightarrow{l_{1}} \cdot \overrightarrow{l_{3}}\right)\left(\overrightarrow{l_{2}} \cdot \overrightarrow{l_{3}}\right)$ leads to $\left(Q_{1}^{3}+6 Q_{1} Q_{2}^{2}+2 Q_{2}^{3}\right) / 9$.
} 


$$
\begin{aligned}
\mathbf{K}_{c}= & Q_{0}\left[4\left(Q_{1} Q_{3}-Q_{2} Q_{3}+3 Q_{2} Q_{4}\right)+\kappa^{2}\left(4 Q_{1} Q_{2}-Q_{1}^{2}-18 Q_{1} Q_{5}-12 Q_{2} Q_{5}+15 Q_{5}^{2}\right)\right] \\
& +12 \kappa^{2} Q_{0}^{2} Q_{4}-\frac{4}{9}\left(10 Q_{1}^{3}+15 Q_{1} Q_{2}^{2}+2 Q_{2}^{3}\right)
\end{aligned}
$$

which allow to compute the next sequence of nine coefficients $\mathcal{N}_{j}$ :

$$
\begin{aligned}
& \mathcal{N}_{16}=2 \int_{0}^{\infty} d r \int_{0}^{\pi / 2} d \varphi\left\{r \mathbf{K}_{a}+32 r s^{2} Q_{0}^{2}\left[Q_{2}+\left(s^{2}+\kappa^{2}\right) Q_{0}\right]\right\}_{\text {reg }}=-2.91600 \\
& \mathcal{N}_{17}=\int_{0}^{\infty} d r \int_{0}^{\pi / 2} d \varphi\left\{\frac{r}{2}\left(4 s^{2} \mathbf{K}_{a}+\mathbf{K}_{b}+\mathbf{K}_{c}\right)+32 r s^{4} Q_{0}^{2}\left[Q_{1}+2 Q_{2}+\left(2 s^{2}+3 \kappa^{2}\right) Q_{0}\right]\right\}_{\mathrm{reg}}=-2.78344 \\
& \mathcal{N}_{18}=\int_{0}^{\infty} d r \int_{0}^{\pi / 2} d \varphi\left\{\frac{r}{2}\left(\mathbf{K}_{b}+\mathbf{K}_{c}-4 s^{2} \mathbf{K}_{a}\right)+32 r s^{4} Q_{0}^{2}\left[Q_{1}-2 Q_{2}-\left(2 s^{2}+\kappa^{2}\right) Q_{0}\right]\right\}_{\mathrm{reg}}=0.422018 \\
& \mathcal{N}_{19}=12 \int_{0}^{\infty} d r \int_{0}^{\pi / 2} d \varphi\left\{r\left(\mathbf{K}_{a}-32 s^{4} Q_{0}^{3}\right)\right\}_{\text {reg }}=10.1541 \\
& \mathcal{N}_{20}=3 \int_{0}^{\infty} d r \int_{0}^{\pi / 2} d \varphi\left\{r\left(4 s^{2} \mathbf{K}_{a}+\mathbf{K}_{c}\right)-64 r s^{4} Q_{0}^{2}\left[Q_{1}+\left(2 s^{2}+\kappa^{2}\right) Q_{0}\right]\right\}_{\text {reg }}=8.05637 \\
& \mathcal{N}_{21}=3 \int_{0}^{\infty} d r \int_{0}^{\pi / 2} d \varphi\left\{r\left(\mathbf{K}_{c}-4 s^{2} \mathbf{K}_{a}\right)+64 r s^{4} Q_{0}^{2}\left[\left(2 s^{2}-\kappa^{2}\right) Q_{0}-Q_{1}\right]\right\}_{\text {reg }}=-0.745696 \\
& \mathcal{N}_{22}=6 \int_{0}^{\infty} d r \int_{0}^{\pi / 2} d \varphi\left\{r \mathbf{K}_{a}+32 r s^{2} Q_{0}^{2}\left[\left(s^{2}-\kappa^{2}\right) Q_{0}-Q_{2}\right]\right\}_{\mathrm{reg}}=-1.07623 \\
& \mathcal{N}_{23}=3 \int_{0}^{\infty} d r \int_{0}^{\pi / 2} d \varphi\left\{\frac{r}{2}\left(4 s^{2} \mathbf{K}_{a}-\mathbf{K}_{b}+\mathbf{K}_{c}\right)+32 r s^{4} Q_{0}^{2}\left[Q_{1}-2 Q_{2}+\left(2 s^{2}-\kappa^{2}\right) Q_{0}\right]\right\}_{\text {reg }}=-3.23779 \\
& \mathcal{N}_{24}=3 \int_{0}^{\infty} d r \int_{0}^{\pi / 2} d \varphi\left\{\frac{r}{2}\left(\mathbf{K}_{c}-\mathbf{K}_{b}-4 s^{2} \mathbf{K}_{a}\right)+32 r s^{4} Q_{0}^{2}\left[Q_{1}+2 Q_{2}+\left(3 \kappa^{2}-2 s^{2}\right) Q_{0}\right]\right\}_{\text {reg }}=1.30198 .
\end{aligned}
$$

Finally, the contributions cubic in the tensor-couplings $t_{4}, t_{5}$ lead to the combinations:

$$
\mathbf{K}_{d}=4 s^{2} Q_{0}^{2}\left[3 Q_{3}-9 Q_{4}+2 \kappa^{2}\left(4 Q_{1}-7 Q_{2}-9 Q_{5}\right)\right]-72 s^{2} Q_{0} Q_{2}^{2},
$$

and

$$
\begin{aligned}
\mathbf{K}_{e}= & 6 Q_{0}\left(Q_{2}+\kappa^{2} Q_{0}\right)\left(Q_{3}-3 Q_{4}\right)+\frac{2}{9}\left(35 Q_{1}^{3}+21 Q_{1} Q_{2}^{2}-2 Q_{2}^{3}\right) \\
& +\kappa^{2} Q_{0}\left(27 Q_{1} Q_{5}-22 Q_{1} Q_{2}+18 Q_{2} Q_{5}+\frac{23}{2} Q_{1}^{2}-\frac{45}{2} Q_{5}^{2}\right)
\end{aligned}
$$

of the euclidean polarization functions which allow us to compute the last four coefficients $\mathcal{N}_{j}$ :

$$
\mathcal{N}_{25}=\frac{2}{9} \int_{0}^{\infty} d r \int_{0}^{\pi / 2} d \varphi\left\{r\left(\mathbf{K}_{d}+\mathbf{K}_{e}\right)-64 r s^{4} Q_{0}^{2}\left[3 Q_{2}+\left(2 s^{2}+3 \kappa^{2}\right) Q_{0}\right]\right\}_{\mathrm{reg}}=0.758472
$$




$$
\begin{aligned}
& \mathcal{N}_{26}=-2 \int_{0}^{\infty} d r \int_{0}^{\pi / 2} d \varphi\left\{\frac{r}{3}\left(\mathbf{K}_{d}+3 \mathbf{K}_{e}\right)+64 r s^{4} Q_{0}^{2}\left[Q_{2}+\left(2 s^{2}+\kappa^{2}\right) Q_{0}\right]\right\}_{\text {reg }}=5.01569 \\
& \mathcal{N}_{27}=2 \int_{0}^{\infty} d r \int_{0}^{\pi / 2} d \varphi\left\{\frac{r}{3}\left(\mathbf{K}_{d}-3 \mathbf{K}_{e}\right)+64 r s^{4} Q_{0}^{2}\left[\left(2 s^{2}-\kappa^{2}\right) Q_{0}-Q_{2}\right]\right\}_{\mathrm{reg}}=-1.83226 \\
& \mathcal{N}_{28}=\frac{10}{3} \int_{0}^{\infty} d r \int_{0}^{\pi / 2} d \varphi\left\{r\left(\mathbf{K}_{d}-\mathbf{K}_{e}\right)+64 r s^{4} Q_{0}^{2}\left[3 Q_{2}+\left(3 \kappa^{2}-2 s^{2}\right) Q_{0}\right]\right\}_{\mathrm{reg}}=5.40146 .
\end{aligned}
$$

One observes that the entire set of the $28 \mathcal{N}_{j}$-coefficients spans in magnitude a range from 0.28 up to 10 and several subgroups are similar in size. In view of the complexity of a four-loop calculation, it is remarkable that all coefficients $\mathcal{N}_{j}$ could be computed with such a high numerical precision.

\section{Third-order ring diagrams with one-pion exchange}

In this section we evaluate the third-order particle-hole ring diagrams shown in Fig. 1 with the longest-range component of the NN-interaction, namely the one-pion exchange. After antisymmetrization the $1 \pi$-exchange potential in momentum space takes the (Galilei-invariant) form:

$$
\begin{aligned}
V_{1 \pi}= & -\frac{g_{A}^{2}}{4 f_{\pi}^{2}}\left\{\vec{\tau}_{1} \cdot \vec{\tau}_{2} \frac{\vec{\sigma}_{1} \cdot\left(\vec{q}_{\text {in }}-\vec{q}_{\text {out }}\right) \vec{\sigma}_{2} \cdot\left(\vec{q}_{\text {in }}-\vec{q}_{\text {out }}\right)}{m_{\pi}^{2}+\left(\vec{q}_{\text {in }}-\vec{q}_{\text {out }}\right)^{2}}\right. \\
& \left.+\frac{1}{4}\left(\vec{\tau}_{1} \cdot \vec{\tau}_{2}-3\right) \frac{2 \vec{\sigma}_{1} \cdot\left(\vec{q}_{\text {in }}+\vec{q}_{\text {out }}\right) \vec{\sigma}_{2} \cdot\left(\vec{q}_{\text {in }}+\vec{q}_{\text {out }}\right)+\left(1-\vec{\sigma}_{1} \cdot \vec{\sigma}_{2}\right)\left(\vec{q}_{\text {in }}+\vec{q}_{\text {out }}\right)^{2}}{m_{\pi}^{2}+\left(\vec{q}_{\text {in }}+\vec{q}_{\text {out }}\right)^{2}}\right\},
\end{aligned}
$$

with $g_{A}=1.29$ the nucleon axial-vector coupling constant, $f_{\pi}=92.2 \mathrm{MeV}$ the pion decay constant, and $m_{\pi}=135 \mathrm{MeV}$ the (neutral) pion mass. The direct diagram $I=d i r^{3}$ is very easily evaluated since the interaction product depends only on the momentum $\vec{q}$ flowing through the three polarization bubbles. The pertinent contribution to the energy per particle of isospin-symmetric nuclear matter reads:

$$
\bar{E}\left(k_{f}\right)^{(\mathrm{I})}=-\frac{3 g_{A}^{6} M^{2} k_{f}^{5}}{32 \pi^{7} f_{\pi}^{6}} \int_{0}^{\infty} d s \int_{0}^{\infty} d \kappa\left[\frac{s^{2} Q_{0}(s, \kappa)}{s^{2}+\beta}\right]^{3}
$$

with the ratio $\beta=m_{\pi}^{2} / 4 k_{f}^{2}$. Note that the expression for $\bar{E}\left(k_{f}\right)^{(\mathrm{I})}$ could also be inferred from the photon-exchange ring energy discussed in textbooks [20, 21]. For the exchange-type diagram $I I=-d i r^{2}$ exc only one internal loop-integral can solved in terms of the polarization function $\Pi(\omega, \vec{q})$ introduced in eq.(5). Putting all the pieces together, one ends up with the following representation of the energy per particle:

$$
\begin{aligned}
\bar{E}\left(k_{f}\right)^{(\mathrm{II})}= & \frac{9 g_{A}^{6} M^{2} k_{f}^{5}}{(2 \pi)^{7} f_{\pi}^{6}} \int_{0}^{\infty} d s \int_{0}^{\infty} d \kappa \int_{0}^{1} d l_{1} \int_{0}^{1} d l_{2} \int_{-l_{1}}^{l_{1}} d x \int_{-l_{2}}^{l_{2}} d y \frac{l_{1} l_{2} s^{4} Q_{0}(s, \kappa)\left(s^{2}+\beta\right)^{-2}}{\left[(s+x)^{2}+\kappa^{2}\right]\left[(s+y)^{2}+\kappa^{2}\right]} \\
& \times\left\{(s+x)(s+y)+\left[2 \beta+(x-y)^{2}\right]\left[\kappa^{2}-(s+x)(s+y)\right] W_{a}^{-1 / 2}\right. \\
& \left.-\left[2 \beta+(2 s+x+y)^{2}\right]\left[\kappa^{2}+(s+x)(s+y)\right] W_{b}^{-1 / 2}\right\},
\end{aligned}
$$


with the radicands $W_{a}=\left[4 \beta+l_{1}^{2}+l_{2}^{2}-2 x y\right]^{2}-4\left(l_{1}^{2}-x^{2}\right)\left(l_{2}^{2}-y^{2}\right)$ and $W_{b}=\left[4 \beta+l_{1}^{2}+l_{2}^{2}+4 s(s+\right.$ $x+y)+2 x y]^{2}-4\left(l_{1}^{2}-x^{2}\right)\left(l_{2}^{2}-y^{2}\right)$. Apparently, the factor $s^{4} Q_{0}(s, \kappa)\left(s^{2}+\beta\right)^{-2}$ in the numerator of eq. (67) originates from the polarization function $\Pi(\omega, \vec{q})$ and two direct $1 \pi$-exchanges depending on $\vec{q}$. For the remaining two exchange-type diagrams $I I I=\operatorname{dir} e x c^{2}$ and $I V=-\operatorname{exc}^{3}$ only the frequency-integral over the energy denominators $i \omega+\overrightarrow{l_{j}} \cdot \vec{q} / M$ from the polarization functions (see eq.(5)) can be performed analytically and residue calculus provides the relevant formula:

$$
\int_{-\infty}^{\infty} \frac{d \omega}{2 \pi} \frac{1}{(i \omega+a)(i \omega+b)(i \omega+c)}=\frac{\theta(a)}{(a-b)(a-c)}+\frac{\theta(b)}{(b-a)(b-c)}+\frac{\theta(c)}{(c-a)(c-b)},
$$

with $a, b, c$ three real-valued parameters. In the end the contributions to the energy per particle, $\bar{E}\left(k_{f}\right)^{(\mathrm{III})}$ and $\bar{E}\left(k_{f}\right)^{(\mathrm{IV})}$, have representations in the form of 9-dimensional integrals over four radii (three from 0 to 1 and one from 0 to $\infty$ ), three directional cosines (from -1 to 1 ) and two azimuthal angles (from 0 to $2 \pi$ ). The expressions for the integrands are very lengthy and not given here.

In pure neutron matter the four ring diagrams generated by $1 \pi$-exchange carry different isospinfactors and the energy per particle is composed as:

$$
\bar{E}_{n}\left(k_{n}\right)=\frac{1}{12} \bar{E}\left(k_{n}\right)^{(\mathrm{I})}-\frac{1}{6} \bar{E}\left(k_{n}\right)^{(\mathrm{II})}+\frac{1}{3} \bar{E}\left(k_{n}\right)^{(\mathrm{III})}+\frac{1}{12} \bar{E}\left(k_{n}\right)^{(\mathrm{IV})} .
$$

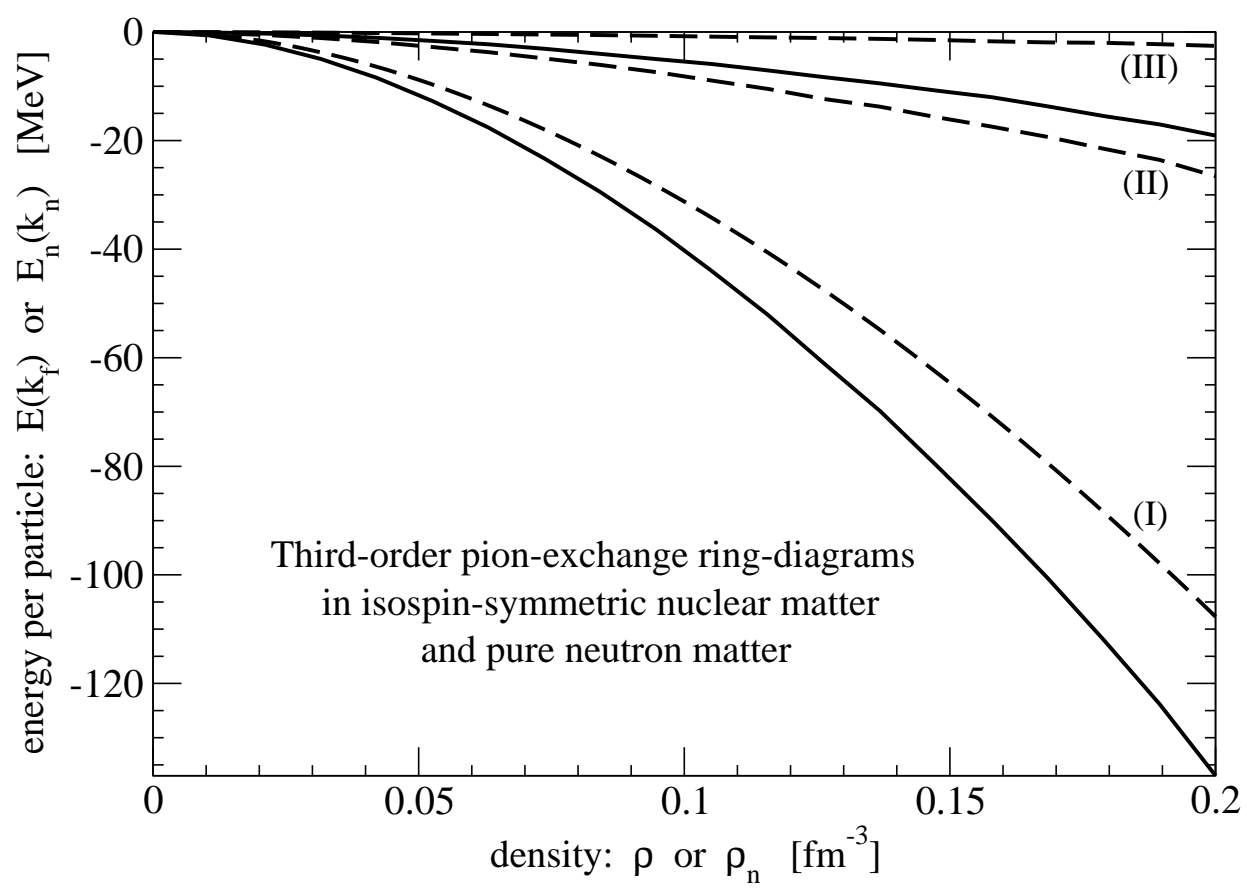

Figure 2: Contributions of the third-order particle-hole ring-diagrams generated by $1 \pi$-exchange to the energy per particle of isospin-symmetric nuclear matter and pure neutron matter.

Fig. 2 shows by the dashed lines the contributions from individual ring diagrams to the energy per particle $\bar{E}\left(k_{f}\right)$ as a function of the density $\rho=2 k_{f}^{3} / 3 \pi^{2}$. The part $I V=-e x c^{3}$ is so small, that it is not visible on the scale of the figure. One observes that the exchange corrections are successively suppressed against the direct term, $|I I I| \ll|I I| \ll|I|$, but they are all of the same attractive sign. At saturation density $\rho_{0}=0.16 \mathrm{fm}^{-3}$ (or $k_{f 0}=263 \mathrm{MeV}$ ) one gets from the thirdorder particle-hole diagrams with long-range $1 \pi$-exchange a tremendous attraction of $\bar{E}\left(k_{f 0}\right)=$ $-92.0 \mathrm{MeV}$. In pure neutron matter at the same density it reduces to $\bar{E}_{n}\left(1.26 k_{f 0}\right)=-12.7 \mathrm{MeV}$. The density-dependence of the pionic three-ring energies is shown by the full lines in Fig. 2. We 
note as an aside that in the chiral limit $m_{\pi}=0$ the pionic three-ring energies scale simply with the fifth power of the Fermi momentum $\left(k_{f}\right.$ or $\left.k_{n}\right)$ :

$$
\bar{E}\left(k_{f}\right)_{m_{\pi}=0}^{3 \pi-\text { ring }} \simeq-\frac{g_{A}^{6} M^{2} k_{f}^{5}}{\left(2 \pi f_{\pi}\right)^{6}} \cdot 0.812, \quad \bar{E}_{n}\left(k_{n}\right)_{m_{\pi}=0}^{3 \pi-\operatorname{ring}} \simeq-\frac{g_{A}^{6} M^{2} k_{n}^{5}}{\left(4 \pi f_{\pi}\right)^{6}} \cdot 2.37 .
$$

At this point it is important to stress that the results presented in Fig. 2 are in no way representative for the third-order particle-hole contributions computed from realistic chiral low-momentum NNpotentials. As one can see from table I in ref. [14] the three-ring attraction in isospin-symmetric nuclear matter is bounded by about $2 \mathrm{MeV}$ at $\rho_{0}=0.16 \mathrm{fm}^{-3}$, when using chiral NN-potentials with soft cutoffs $\Lambda<500 \mathrm{MeV}$. Nevertheless the semi-analytical approach developed here is very helpful in order to benchmark the extensive numerical computations [8] based on the partial-wave decomposition of the NN-potential for test-interactions of the one-boson exchange type [14]. Taking for example a pure spin-orbit interaction $\sim i\left(\vec{\sigma}_{1}+\vec{\sigma}_{2}\right) \cdot\left(\vec{q}_{\text {out }} \times \vec{q}_{\text {in }}\right)$, one finds that the third-order ring diagrams vanish identically. In the semi-analytical approach this property follows immediately from the triple spin-trace being equal to zero, whereas in the partial-wave based method delicate cancelations in multiple partial-wave sums are at work.

It is also interesting to consider a direct NN-contact interaction $V_{\text {dir }}=-C \vec{\tau}_{1} \cdot \vec{\tau}_{2} \vec{\sigma}_{1} \cdot\left(\vec{q}_{\text {in }}-\right.$ $\left.\vec{q}_{\text {out }}\right) \vec{\sigma}_{2} \cdot\left(\vec{q}_{\text {in }}-\vec{q}_{\text {out }}\right)$ with the same isospin- and spin-dependence as $1 \pi$-exchange. Evaluating the four diagrams in Fig. 1 and applying dimensional regularization as done in subsection 3.1, one finds for the energy per particle:

$$
\begin{gathered}
\bar{E}\left(k_{f}\right)=C^{3} M^{2} k_{f}^{11} \pi^{-7}(5.73956+0.248478+0.186314+0)=C^{3} M^{2} k_{f}^{11} \pi^{-7} \cdot 6.17435 \\
\bar{E}_{n}\left(k_{n}\right)=C^{3} M^{2} k_{n}^{11} \pi^{-7} \cdot 0.498988
\end{gathered}
$$

It is astonishing that the diagram $I V=-e x c^{3}$ vanishes identically for $V_{\text {dir }}=-C \vec{\tau}_{1} \cdot \vec{\tau}_{2} \vec{\sigma}_{1} \cdot\left(\vec{q}_{\text {in }}-\right.$ $\left.\vec{q}_{\text {out }}\right) \vec{\sigma}_{2} \cdot\left(\vec{q}_{\text {in }}-\vec{q}_{\text {out }}\right)$. In the actual calculation 43 terms had to be translated into cubic expressions in $Q_{j}(s, \kappa)$, which in the end summed up to zero. This fact gives also an explanation for the observed smallness of the contribution $I V=-e x c^{3}$ when evaluated with the long-range $1 \pi$-exchange.

\section{$6 \quad$ Third-order ladder diagrams}
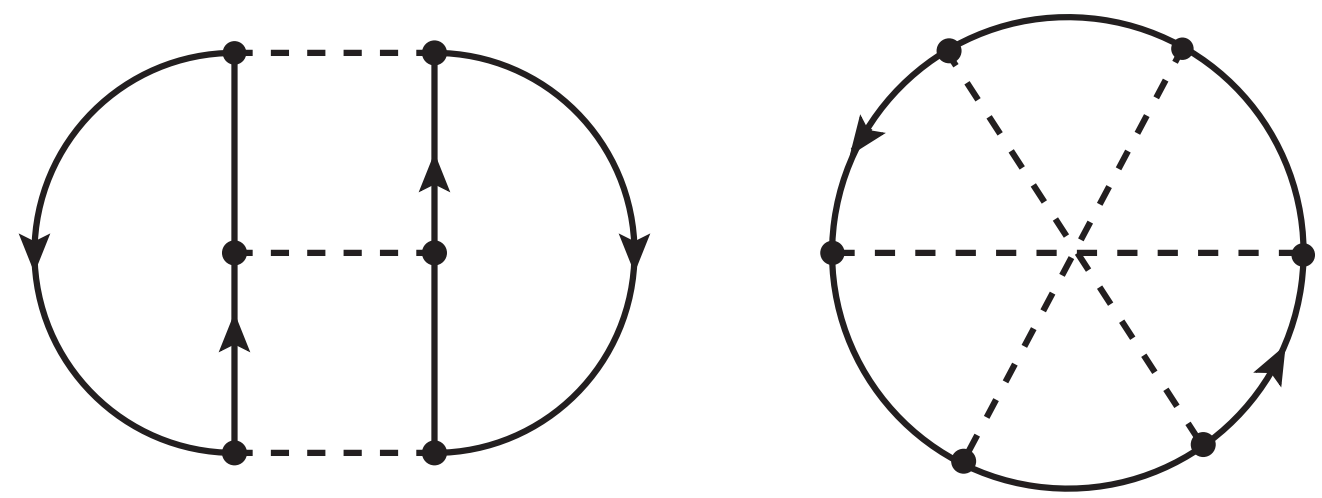

Figure 3: Third order ladder diagrams separated into direct and exchange contributions.

In this section we return to the general $\mathcal{O}\left(p^{2}\right)$ NN-contact interaction and calculate the remaining third-order contributions arising from (particle-particle and hole-hole) ladder diagrams. Fig. 3 shows the direct and exchange-type ladder diagrams at third-order with the two-body interaction symbolized by a dashed line. We employ the techniques of refs. [22, 23] based on the complex-valued in-medium loop, which keeps together particle-particle and hole-hole ladder contributions. 
We start with the Skyrme NN-contact interaction $V_{\mathrm{Sk}}$ which involves two s-wave terms $\sim t_{0}, t_{1}$ and two p-wave terms $\sim t_{2}, W_{0}$. As shown in appendix $\mathrm{A}$ of ref.[17], interferences of s-wave and p-wave interactions (in ladder diagrams) vanish in a medium with one single Fermi-momentum (see also eqs. $(5,6)$ in ref.[23]). The case of pure neutron matter is particularly simple, because two neutrons interact either in the (odd) ${ }^{1} S_{0}$-state or the (even) ${ }^{3} P$-states. Taking into account all $\left(t_{0} t_{1}\right.$ and $\left.t_{2} W_{0}\right)$ interference terms possible at third order, one finds for the energy per particle:

$$
\begin{aligned}
\bar{E}_{n}\left(k_{n}\right)^{(3-\mathrm{lad})}= & \frac{M^{2} k_{n}^{5}}{64 \pi^{6}}\left\{t_{0}^{3}\left(1-x_{0}\right)^{3} \mathcal{B}_{1}+k_{n}^{2} t_{0}^{2} t_{1}\left(1-x_{0}\right)^{2}\left(1-x_{1}\right) \mathcal{B}_{2}+k_{n}^{4} t_{0} t_{1}^{2}\left(1-x_{0}\right)\left(1-x_{1}\right)^{2} \mathcal{B}_{3}\right. \\
& \left.+k_{n}^{6} t_{1}^{3}\left(1-x_{1}\right)^{3} \mathcal{B}_{4}+k_{n}^{6} t_{2}^{3}\left(1+x_{2}\right)^{3} \mathcal{B}_{5}+k_{n}^{6} t_{2}\left(1+x_{2}\right) W_{0}^{2} \mathcal{B}_{6}+k_{n}^{6} W_{0}^{3} \mathcal{B}_{7}\right\}
\end{aligned}
$$

with seven coefficients $\mathcal{B}_{j}$ to be determined later. The result for isospin-symmetric nuclear matter takes a similar form:

$$
\begin{aligned}
\bar{E}\left(k_{f}\right)^{(3-\mathrm{lad})}= & \frac{M^{2} k_{f}^{5}}{64 \pi^{6}}\left\{3 t_{0}^{3}\left(1+3 x_{0}^{2}\right) \mathcal{B}_{1}+3 k_{f}^{2} t_{0}^{2} t_{1}\left(1+x_{0}^{2}+2 x_{0} x_{1}\right) \mathcal{B}_{2}\right. \\
& +3 k_{f}^{4} t_{0} t_{1}^{2}\left(1+2 x_{0} x_{1}+x_{1}^{2}\right) \mathcal{B}_{3}+3 k_{f}^{6} t_{1}^{3}\left(1+3 x_{1}^{2}\right) \mathcal{B}_{4} \\
& \left.+k_{f}^{6} t_{2}^{3}\left(\frac{5}{3}+4 x_{2}+5 x_{2}^{2}+\frac{4 x_{2}^{3}}{3}\right) \mathcal{B}_{5}+\frac{3 k_{f}^{6}}{2} t_{2}\left(1+x_{2}\right) W_{0}^{2} \mathcal{B}_{6}+\frac{3 k_{f}^{6}}{2} W_{0}^{3} \mathcal{B}_{7}\right\}
\end{aligned}
$$

where the different dependences on $x_{0}$ and $x_{1}$ follow from summing the weights in the isotriplet ${ }^{1} S_{0}$-state and the isosinglet ${ }^{3} S_{1}$-state: $\left[3\left(1-x_{0}\right)^{3}+3\left(1+x_{0}\right)^{3}\right] / 2=3 t_{0}^{3}\left(1+3 x_{0}^{2}\right)$ and $\left[3\left(1-x_{0}\right)^{2}(1-\right.$ $\left.\left.x_{1}\right)+3\left(1+x_{0}\right)^{2}\left(1+x_{1}\right)\right] / 2=3\left(1+x_{0}^{2}+2 x_{0} x_{1}\right)$. The factor $1 / 2$ comes here from dividing energy densities by particle densities. For the p-wave term $\sim t_{2}^{3}$ the reweighting goes as $9\left(1+x_{2}\right)^{3} \rightarrow$ $\left[3\left(1-x_{2}\right)^{3}+27\left(1+x_{2}\right)^{3}\right] / 2=3\left(5+12 x_{2}+15 x_{2}^{2}+4 x_{2}^{3}\right)$. For the interactions $\sim t_{2}\left(1+x_{2}\right), W_{0}$ which operate only in isotriplet ${ }^{3} P$-states, one can deduce from a comparison of the energy density in pure neutron matter and isospin-symmetric nuclear matter the relation, $3($ Hart + Fock $)=4$ Hart +2 Fock, between the (double) spin-trace of the direct diagram (Hart) and the (single) spin-trace of the exchange-type diagram (Fock). Consequently, one has Hart = Fock and this explains the relative factor $3 / 2$ for the last two terms in eqs. $(73,74)$.

In order to compute the coefficients $\mathcal{B}_{j}$ one needs the in-medium loop, whose real and imaginary part read [22, 23]:

$$
\begin{gathered}
R(s, \kappa)=2+\frac{1}{2 s}\left[1-(s+\kappa)^{2}\right] \ln \frac{1+s+\kappa}{|1-s-\kappa|}+\frac{1}{2 s}\left[1-(s-\kappa)^{2}\right] \ln \frac{1+s-\kappa}{1-s+\kappa}, \\
I(s, \kappa)=\kappa \theta(1-s-\kappa)+\frac{1}{2 s}\left(1-s^{2}-\kappa^{2}\right) \theta(s+\kappa-1) .
\end{gathered}
$$

Furthermore, iterated p-wave contact-interactions introduce a factor $l_{i} l_{j}$ and the corresponding tensorial in-medium loop is decomposed into a transversal projector $\delta_{i j}-\hat{P}_{i} \hat{P}_{j}$ and a longitudinal projector $\hat{P}_{i} \hat{P}_{j}$, where $\vec{P}=\left(\vec{p}_{1}+\vec{p}_{2}\right) / 2$ with $\left|\vec{p}_{1,2}\right|<k_{f}$ (for details see section 3 in ref. [23]). The transversal in-medium loop has the following real and imaginary part [23]:

$$
\begin{aligned}
R_{\perp}(s, \kappa)= & \frac{2}{3}-\frac{s^{2}}{4}+2 \kappa^{2}+\frac{\left(1-\kappa^{2}\right)^{2}}{4 s^{2}}+\frac{\left[(s+\kappa)^{2}-1\right]^{2}}{16 s^{3}}\left(s^{2}+\kappa^{2}-4 s \kappa-1\right) \\
& \times \ln \frac{1+s+\kappa}{|1-s-\kappa|}+\frac{\left[(s-\kappa)^{2}-1\right]^{2}}{16 s^{3}}\left(s^{2}+\kappa^{2}+4 s \kappa-1\right) \ln \frac{1+s-\kappa}{1-s+\kappa}, \\
I_{\perp}(s, \kappa)=\kappa^{3} \theta & \theta(1-s-\kappa)+\frac{1-s^{2}-\kappa^{2}}{16 s^{3}}\left[12 s^{2} \kappa^{2}-\left(1-s^{2}-\kappa^{2}\right)^{2}\right] \theta(s+\kappa-1),
\end{aligned}
$$


while the real and imaginary part of the longitudinal in-medium loop read [23]:

$$
\begin{aligned}
R_{\|}(s, \kappa)= & \frac{8}{3}+\frac{s^{2}}{2}+2 \kappa^{2}-\frac{\left(1-\kappa^{2}\right)^{2}}{2 s^{2}}+\left[\frac{\left(1-s^{2}-\kappa^{2}\right)^{3}}{8 s^{3}}-\kappa^{3}\right] \\
& \times \ln \frac{1+s+\kappa}{|1-s-\kappa|}+\left[\frac{\left(1-s^{2}-\kappa^{2}\right)^{3}}{8 s^{3}}+\kappa^{3}\right] \ln \frac{1+s-\kappa}{1-s+\kappa}, \\
I_{\|}(s, \kappa) & =\kappa^{3} \theta(1-s-\kappa)+\frac{\left(1-s^{2}-\kappa^{2}\right)^{3}}{8 s^{3}} \theta(s+\kappa-1) .
\end{aligned}
$$

Note that these functions satisfy the relations: $2 R_{\perp}(s, \kappa)+R_{\|}(s, \kappa)=4+3 \kappa^{2} R(s, \kappa)$ and $2 I_{\perp}(s, \kappa)+$ $I_{\|}(s, \kappa)=3 \kappa^{2} I(s, \kappa)$.

Now we can specify the formulas for the seven four-loop coefficients $\mathcal{B}_{j}, j=1, \ldots, 7$ together with their numerical values:

$$
\mathcal{B}_{1}=8 \int_{0}^{1} d s s^{2} \int_{0}^{\sqrt{1-s^{2}}} d \kappa \kappa I(s, \kappa)\left[3 R^{2}(s, \kappa)-\pi^{2} I^{2}(s, \kappa)\right]=1.1716223,
$$

which has also been quoted in the right part of eq.(1). The detailed derivation that $R^{2}-\pi^{2} I^{2} / 3$ is the correct expression for the combined third-order ladder contribution has been presented in section 4 of ref.[22]. From now on we will drop the arguments $s$ and $\kappa$ :

$$
\begin{aligned}
& \mathcal{B}_{2}=8 \int_{0}^{1} d s s^{2} \int_{0}^{\sqrt{1-s^{2}}} d \kappa \kappa I\left[3 \kappa^{2}\left(3 R^{2}-\pi^{2} I^{2}\right)+8 R\right]=1.9893144 \\
& \mathcal{B}_{3}=8 \int_{0}^{1} d s s^{2} \int_{0}^{\sqrt{1-s^{2}}} d \kappa \kappa I\left[3 \kappa^{4}\left(3 R^{2}-\pi^{2} I^{2}\right)+\left(2 s^{2}+14 \kappa^{2}+\frac{6}{5}\right) R+4\right]=1.360736 \\
& \mathcal{B}_{4}=8 \int_{0}^{1} d s s^{2} \int_{0}^{\sqrt{1-s^{2}}} d \kappa \kappa I\left[\kappa^{6}\left(3 R^{2}-\pi^{2} I^{2}\right)+2 \kappa^{2}\left(s^{2}+3 \kappa^{2}+\frac{3}{5}\right) R+\frac{4 s^{2}}{3}+\frac{8 \kappa^{2}}{3}+\frac{4}{5}\right]=0.3344923 \\
& \mathcal{B}_{5}=\frac{8}{9} \int_{0}^{1} d s s^{2} \int_{0}^{\sqrt{1-s^{2}}} d \kappa \kappa\left[2 I_{\perp}\left(3 R_{\perp}^{2}-\pi^{2} I_{\perp}^{2}\right)+I_{\|}\left(3 R_{\|}^{2}-\pi^{2} I_{\|}^{2}\right)\right]=0.06699116 \\
& \mathcal{B}_{6}=\frac{128}{9} \int_{0}^{1} d s s^{2} \int_{0}^{\sqrt{1-s^{2}}} d \kappa \kappa\left\{I_{\perp}\left[3 R_{\perp}^{2}+2 R_{\perp} R_{\|}+R_{\|}^{2}-\frac{\pi^{2}}{3}\left(3 I_{\perp}^{2}+2 I_{\perp} I_{\|}+I_{\|}^{2}\right)\right]\right. \\
& \left.+I_{\|}\left[R_{\perp}\left(R_{\perp}+2 R_{\|}\right)-\frac{\pi^{2}}{3} I_{\perp}\left(I_{\perp}+2 I_{\|}\right)\right]\right\}=1.327456, \\
& \mathcal{B}_{7}=\frac{128}{9} \int_{0}^{1} d s s^{2} \int_{0}^{\sqrt{1-s^{2}}} d \kappa \kappa\left\{2 I_{\perp}\left(R_{\perp} R_{\|}-\frac{\pi^{2}}{3} I_{\perp} I_{\|}\right)+I_{\|}\left(R_{\perp}^{2}-\frac{\pi^{2}}{3} I_{\perp}^{2}\right)\right\}=0.4527642 .
\end{aligned}
$$

It is worth to note that $I(s, \kappa)$ and $I_{\perp, \|}(s, \kappa)$ serve also as weight-functions for the reduction of six-dimensional integrals over two Fermi spheres $\left|\vec{p}_{1,2}\right|<k_{f}$ to double-integrals $\int_{0}^{1} d s s^{2} \int_{0}^{\sqrt{1-s^{2}}} d \kappa \kappa$. 
Next, we come to the additional third-order ladder contributions generated by the tensor contact-interaction $V_{\text {ten }}$. One finds for the energy per particle of pure neutron matter the result:

$$
\bar{E}_{n}\left(k_{n}\right)^{(3-\mathrm{lad})}=\frac{M^{2} k_{n}^{11}}{64 \pi^{6}}\left\{t_{5} W_{0}^{2} \mathcal{B}_{8}+t_{5}^{2} t_{2}\left(1+x_{2}\right) \mathcal{B}_{9}+t_{5}^{2} W_{0} \mathcal{B}_{10}+t_{5}^{3} \mathcal{B}_{11}\right\}
$$

which depends only the new parameter $t_{5}$. Further p-wave interference terms proportional to $t_{5} t_{2}^{2}$ and $t_{5} t_{2} W_{0}$ are absent because of vanishing spin-traces. Since the tensor term $\sim t_{5}$ acts only in isovector ${ }^{3} P$-states the expression in eq.(88) reappears in the energy per particle of isospinsymmetric nuclear matter:

$$
\begin{aligned}
\bar{E}\left(k_{f}\right)^{(3-\mathrm{lad})}= & \frac{M^{2} k_{f}^{11}}{64 \pi^{6}}\left\{\frac{3}{2} t_{5} W_{0}^{2} \mathcal{B}_{8}+\frac{3}{2} t_{5}^{2} t_{2}\left(1+x_{2}\right) \mathcal{B}_{9}+\frac{3}{2} t_{5}^{2} W_{0} \mathcal{B}_{10}\right. \\
& \left.+\frac{3}{2} t_{5}^{3} \mathcal{B}_{11}+k_{f}^{-2} t_{4}^{2} t_{0}\left(1+x_{0}\right) \mathcal{B}_{12}+t_{4}^{2} t_{1}\left(1+x_{1}\right) \mathcal{B}_{13}\right\}
\end{aligned}
$$

with a factor $3 / 2$. The last two terms $\sim t_{4}^{2}$ in eq.(89) are produced by ${ }^{3} S_{1-}{ }^{3} D_{1}$ mixing at second order. It has to be combined at third-order with an s-wave interaction in the ${ }^{3} S_{1}$-channel, which determines the parameter combinations $t_{0,1}\left(1+x_{0,1}\right)$. Note that for third-order interference terms all possible orderings need to be considered, since in general these lead to different spin-traces.

Finally, we specify the formulas for the remaining six four-loop coefficients $\mathcal{B}_{j}, j=8, \ldots, 13$ together with their numerical values:

$$
\begin{aligned}
& \mathcal{B}_{8}=-\frac{256}{27} \int_{0}^{1} d s s^{2} \int_{0}^{\sqrt{1-s^{2}}} d \kappa \kappa\left\{I_{\perp}\left[3 R_{\perp}^{2}+11 R_{\perp} R_{\|}+R_{\|}^{2}-\frac{\pi^{2}}{3}\left(3 I_{\perp}^{2}+11 I_{\perp} I_{\|}+I_{\|}^{2}\right)\right]\right. \\
& \left.+I_{\|}\left[R_{\perp}\left(\frac{11}{2} R_{\perp}+2 R_{\|}\right)-\frac{\pi^{2}}{6} I_{\perp}\left(11 I_{\perp}+4 I_{\|}\right)\right]\right\}=-2.243263, \\
& \mathcal{B}_{9}=\frac{128}{9} \int_{0}^{1} d s s^{2} \int_{0}^{\sqrt{1-s^{2}}} d \kappa \kappa\left\{I_{\perp}\left[7 R_{\perp}^{2}+2 R_{\perp} R_{\|}+R_{\|}^{2}-\frac{\pi^{2}}{3}\left(7 I_{\perp}^{2}+2 I_{\perp} I_{\|}+I_{\|}^{2}\right)\right]\right. \\
& \left.+I_{\|}\left[R_{\perp}^{2}+2 R_{\perp} R_{\|}+2 R_{\|}^{2}-\frac{\pi^{2}}{3}\left(I_{\perp}^{2}+2 I_{\perp} I_{\|}+2 I_{\|}^{2}\right)\right]\right\}=2.042028, \\
& \mathcal{B}_{10}=\frac{256}{9} \int_{0}^{1} d s s^{2} \int_{0}^{\sqrt{1-s^{2}}} d \kappa \kappa\left\{I_{\perp}\left[6 R_{\perp}^{2}+7 R_{\perp} R_{\|}+2 R_{\|}^{2}-\frac{\pi^{2}}{3}\left(6 I_{\perp}^{2}+7 I_{\perp} I_{\|}+2 I_{\|}^{2}\right)\right]\right. \\
& \left.+I_{\|}\left[R_{\perp}\left(\frac{7}{2} R_{\perp}+4 R_{\|}\right)-\frac{\pi^{2}}{6} I_{\perp}\left(7 I_{\perp}+8 I_{\|}\right)\right]\right\}=6.66812 \\
& \mathcal{B}_{11}=-\frac{256}{81} \int_{0}^{1} d s s^{2} \int_{0}^{\sqrt{1-s^{2}}} d \kappa \kappa\left\{I_{\perp}\left[17 R_{\perp}^{2}+15 R_{\perp} R_{\|}+3 R_{\|}^{2}-\pi^{2}\left(\frac{17}{3} I_{\perp}^{2}+5 I_{\perp} I_{\|}+I_{\|}^{2}\right)\right]\right. \\
& \left.+I_{\|}\left[\frac{15}{2} R_{\perp}^{2}+6 R_{\perp} R_{\|}+4 R_{\|}^{2}-\pi^{2}\left(\frac{5}{2} I_{\perp}^{2}+2 I_{\perp} I_{\|}+\frac{4}{3} I_{\|}^{2}\right)\right]\right\}=-1.655323
\end{aligned}
$$




$$
\begin{aligned}
\mathcal{B}_{12}= & 32 \int_{0}^{1} d s s^{2} \int_{0}^{\sqrt{1-s^{2}}} d \kappa \kappa\left\{I_{\|}\left[R\left(R_{\|}-R_{\perp}\right)-\frac{\pi^{2}}{3} I\left(I_{\|}-I_{\perp}\right)\right]+I\left[8 R\left(\frac{s^{2}-2 \kappa^{2}}{3}+\frac{1}{5}\right)-\frac{8}{3}\right.\right. \\
+ & \left.\left.\frac{\kappa^{4}}{2}\left(\pi^{2} I^{2}-3 R^{2}\right)+R_{\perp}^{2}-\frac{\pi^{2}}{3} I_{\perp}^{2}+\frac{1}{2} R_{\|}^{2}-\frac{\pi^{2}}{6} I_{\|}^{2}+\kappa^{2}\left(3 R R_{\perp}-\pi^{2} I I_{\perp}\right)\right]\right\}=2.421103, \\
\mathcal{B}_{13}= & 32 \int_{0}^{1} d s s^{2} \int_{0}^{\sqrt{1-s^{2}}} d \kappa \kappa\left\{I_{\|}\left[\frac{4}{9}\left(3 s^{2} R+R_{\|}-R_{\perp}\right)+\kappa^{2} R\left(R_{\|}-R_{\perp}\right)-\kappa^{2} \frac{\pi^{2}}{3} I\left(I_{\|}-I_{\perp}\right)\right]\right. \\
& +I\left[4 \kappa^{2}\left(\frac{1}{5}-\kappa^{2}\right) R-\frac{8 \kappa^{2}}{3}+\frac{4}{3} R_{\|}\left(s^{2}+\frac{1}{5}\right)+\frac{4}{3} R_{\perp}\left(\kappa^{2}+\frac{2}{5}\right)+\kappa^{2}\left(R_{\perp}^{2}-\frac{\pi^{2}}{3} I_{\perp}^{2}\right)\right. \\
& \left.\left.+\frac{\kappa^{2}}{2}\left(R_{\|}^{2}-\frac{\pi^{2}}{3} I_{\|}^{2}\right)+\kappa^{4}\left(3 R R_{\perp}-\pi^{2} I I_{\perp}\right)+\frac{\kappa^{6}}{2}\left(\pi^{2} I^{2}-3 R^{2}\right)\right]\right\}=1.559127 .
\end{aligned}
$$

One observes that most of the four-loop coefficients $\mathcal{B}_{j}$ are of similar size.

\section{Appendix: Isospin-asymmetry energy}

In this appendix we evaluate a specific contribution from third-order particle-hole ring diagrams to the isospin-asymmetry energy $A\left(k_{f}\right)$. This density-dependent quantity is defined by the expansion of the energy per particle of isospin-asymmetric nuclear matter, $\bar{E}_{\text {as }}\left(k_{p}, k_{n}\right)=\bar{E}\left(k_{f}\right)+\delta^{2} A\left(k_{f}\right)+$ $\mathcal{O}\left(\delta^{4}\right)$, with the proton/neutron Fermi momenta set to $k_{p, n}=k_{f}(1 \mp \delta)^{1 / 3}$. The considered two-body interaction is a momentum-independent NN-contact interaction:

$$
V_{\mathrm{ct}}=-\frac{\pi}{M}\left[a_{s}+3 a_{t}+\vec{\sigma}_{1} \cdot \vec{\sigma}_{2}\left(a_{t}-a_{s}\right)\right]
$$

parametrized through the spin-singlet and spin-triplet scattering lengths, $a_{s}=M\left(x_{0}-1\right) / 4 \pi$ and $a_{t}=-M\left(1+x_{0}\right) / 4 \pi$. Since $V_{\text {ct }}$ does not change the isospin, the evaluation of the four ring diagrams in Fig. 1 proceeds by distinguishing the cases where closed lines represent protons or neutrons. After performing the (multiple) spin-traces one obtains from the sum of the ring diagrams $I+I I+I I+I V$ an integrand for the energy density of isospin-asymmetric nuclear matter proportional to:

$$
\begin{aligned}
& \frac{1}{6}\left[\left(3 a_{t}+a_{s}\right)^{3}+3\left(a_{t}-a_{s}\right)^{3}\right]\left(\Pi_{p p}+\Pi_{n n}\right)^{3}-\left[16 a_{t}^{3}+\left(a_{s}-a_{t}\right)^{3}\right]\left(\Pi_{p p}+\Pi_{n n}\right)\left(\Pi_{p p}^{2}+\Pi_{n n}^{2}\right) \\
& +\left[16 a_{t}^{3}-\left(a_{s}+a_{t}\right)^{3}\right]\left(\Pi_{p p}^{3}+\Pi_{n n}^{3}\right)-\frac{1}{3}\left[\left(3 a_{t}+a_{s}\right)^{3}-12 a_{t}^{2}\left(3 a_{s}+a_{t}\right)\right]\left(\Pi_{p p}^{3}+\Pi_{p n}^{3}+\Pi_{n p}^{3}+\Pi_{n n}^{3}\right),
\end{aligned}
$$

where the symmetry factors $1 / 6,1 / 2,1 / 2,1 / 6$ and relative signs are already included. The expansion of the (diagonal) polarization functions $\Pi_{n n, p p}(\omega, \vec{q})$ in the isospin-asymmetry parameter $\delta$ takes the form (setting $|\vec{q}|=2 s k_{f}$ and $\left.\omega=2 s \kappa k_{f}^{2} / M\right)$ :

$$
\Pi_{n n, p p}(\omega, \vec{q})=\frac{M k_{f}}{4 \pi^{2} s}\left\{Q_{0}(s, \kappa) \pm \frac{\delta}{6} Q_{0}^{\prime}(s, \kappa)+\frac{\delta^{2}}{36} Q_{0}^{\prime \prime}(s, \kappa)+\ldots\right\},
$$

with the linear and quadratic correction functions:

$$
Q_{0}^{\prime}(s, \kappa)=\ln \frac{(1+s)^{2}+\kappa^{2}}{(1-s)^{2}+\kappa^{2}},
$$




$$
Q_{0}^{\prime \prime}(s, \kappa)=\frac{4 s\left(s^{2}+\kappa^{2}-1\right)}{\left[(1+s)^{2}+\kappa^{2}\right]\left[(1-s)^{2}+\kappa^{2}\right]}-\ln \frac{(1+s)^{2}+\kappa^{2}}{(1-s)^{2}+\kappa^{2}} .
$$

The $p n$-mixed polarization function introduced by the diagram $I V=-e x c^{3}$ has the following representation supplemented by an expansion in powers of $\delta / 6$ :

$$
\begin{aligned}
\Pi_{p n}(\omega, \vec{q})=\Pi_{n p}^{*}(\omega, \vec{q}) & =\int \frac{d^{3} l}{(2 \pi)^{3}} \frac{\theta\left(k_{n}-|\vec{l}-\vec{q} / 2|\right)-\theta\left(k_{p}-|\vec{l}+\vec{q} / 2|\right)}{i \omega+\left(k_{n}^{2}-k_{p}^{2}+2 \vec{l} \cdot \vec{q}\right) / 2 M} \\
& =\frac{M k_{f}}{4 \pi^{2} s}\left\{Q_{0}(s, \kappa)-i \frac{\delta}{6} \widetilde{Q}_{0}(s, \kappa)+\frac{\delta^{2}}{36} \widetilde{Q}_{0}(s, \kappa)+\ldots\right\}
\end{aligned}
$$

One recognizes the difference of neutron and proton chemical potentials $\left(k_{n}^{2}-k_{p}^{2}\right) / 2 M$ in the energy denominator. The pertinent linear and quadratic correction functions read:

$$
\begin{gathered}
\widetilde{Q}_{0}(s, \kappa)=\frac{\kappa}{s} \ln \frac{(1+s)^{2}+\kappa^{2}}{(1-s)^{2}+\kappa^{2}}, \\
\widetilde{\widetilde{Q}}_{0}(s, \kappa)=\frac{4\left[\left(1-s^{2}\right)^{2}+\left(3+s^{2}\right) \kappa^{2}\right]}{s\left[(1+s)^{2}+\kappa^{2}\right]\left[(1-s)^{2}+\kappa^{2}\right]}-\left(1+\frac{1}{s^{2}}\right) \ln \frac{(1+s)^{2}+\kappa^{2}}{(1-s)^{2}+\kappa^{2}},
\end{gathered}
$$

Inserting all these ingredients into eq.(97) and expanding up to order $\delta^{2}$, one obtains for the energy per particle:

$$
\bar{E}\left(k_{f}\right)=1.0481446 \frac{\left(a_{s}+a_{t}\right) k_{f}^{5}}{\pi^{4} M}\left(5 a_{s}^{2}+5 a_{t}^{2}-14 a_{s} a_{t}\right),
$$

with the numerical coefficient $\mathcal{N}_{1} / 4=1.0481446$ (see eq.(15)). At the same time the three-ring contribution to the isospin-asymmetry energy $A\left(k_{f}\right) \sim k_{f}^{5}$ comes out as:

$$
A\left(k_{f}\right)=\frac{k_{f}^{5}}{\pi^{4} M}\left(3.5124 a_{s}^{3}+11.092 a_{s}^{2} a_{t}+10.137 a_{s} a_{t}^{2}-5.1014 a_{t}^{3}\right) .
$$

The numerical coefficients stem from evaluating the following (convergent) double-integrals:

$$
\begin{aligned}
& \frac{1}{12} \int_{0}^{\infty} d s \int_{0}^{\infty} d \kappa Q_{0}\left(14 Q_{0} Q_{0}^{\prime \prime}+Q_{0} \widetilde{\widetilde{Q}}_{0}+6 Q_{0}^{\prime 2}-\widetilde{Q}_{0}^{2}\right)=3.5124 \\
& \frac{1}{4} \int_{0}^{\infty} d s \int_{0}^{\infty} d \kappa Q_{0}\left(3 Q_{0} \widetilde{\widetilde{Q}}_{0}-12 Q_{0} Q_{0}^{\prime \prime}+4 Q_{0}^{\prime 2}-3 \widetilde{Q}_{0}^{2}\right)=11.092 \\
& \frac{1}{4} \int_{0}^{\infty} d s \int_{0}^{\infty} d \kappa Q_{0}\left(2 Q_{0}^{\prime 2}+3 \widetilde{Q}_{0}^{2}-6 Q_{0} Q_{0}^{\prime \prime}-3 Q_{0} \widetilde{\widetilde{Q}}_{0}\right)=10.137 \\
& \frac{5}{4} \int_{0}^{\infty} d s \int_{0}^{\infty} d \kappa Q_{0}\left(Q_{0} \widetilde{\widetilde{Q}}_{0}-\widetilde{Q}_{0}^{2}\right)=-5.1014
\end{aligned}
$$

When continuing the expansions in eqs. $(97,98,101)$ up to order $\delta^{4}$, one encounters for the quartic isospin-asymmetry energy a coefficient that is represented by a logarithmically divergent integral. This feature indicates that third-order particle-hole ring diagrams generate the non-analytical term $\delta^{4} \ln |\delta|$ in the isospin-asymmetry expansion, as it has first been found in a second-order calculation with $V_{\text {ct }}$ in ref. 24]. 


\section{Acknowledgements}

I thank S. Petschauer for support in some calculations and J.W. Holt for informative discussions.

\section{References}

[1] E. Epelbaum, Prog. Part. Nucl. Phys. 57, 654 (2006); and refs. therein.

[2] E. Epelbaum, H.W. Hammer and U.G. Meißner, Rev. Mod. Phys. 81, 1773 (2009).

[3] R. Machleidt and D.R. Entem, Phys. Reports 503, 1 (2011); and refs. therein.

[4] I. Tews, T. Krüger, K. Hebeler and A. Schwenk, Phys. Rev. Lett. 110, 032504 (2013).

[5] T. Krüger, I. Tews, K. Hebeler and A. Schwenk, Phys. Rev. C88, 025802 (2013).

[6] C. Drischler, K. Hebeler and A. Schwenk, Phys. Rev. C93, 054314 (2016).

[7] C. Wellenhofer, J.W. Holt, N. Kaiser and W. Weise, Phys. Rev. C89, 064009 (2014).

[8] F. Sammarruca, L. Coraggio, J.W. Holt, N. Itaco, R. Machleidt and L.E. Marcucci, Phys. Rev. C91, 054311 (2015); and refs. therein.

[9] J.W. Holt, N. Kaiser and W. Weise, Prog. Part. Nucl. Phys. 73, 35 (2013).

[10] A. Carbone, A. Rios and A. Polls, Phys. Rev. C88, 044302 (2016).

[11] C. Drischler, A. Carbone, K. Hebeler and A. Schwenk, Phys. Rev. C94, 054306 (2016).

[12] F. Isaule, H.F. Arellano and A. Rios, Phys. Rev. C94, 034004 (2016); and refs. therein.

[13] J.E. Lynn, I. Tews, J. Carlson, S. Gandolfi, A. Gezerlis, K.E. Schmidt and A. Schwenk, Phys. Rev. Lett. 116, 062501 (2016); and refs. therein.

[14] J.W. Holt and N. Kaiser, arXiv:1612.04309 [nucl-th]; and refs. therein.

[15] M. Bender, P.H. Heenen and P.G. Reinhard, Rev. Mod. Phys. 75, 121 (2001).

[16] J.R. Stone and P.G. Reinhard, Prog. Part. Nucl. Phys. 58, 587 (2007).

[17] N. Kaiser, J. Phys. G: Nucl. Part. Phys. 42, 095111 (2015); and refs. therein.

[18] K. Moghrabi, arXiv:1607.05829 [nucl-th].

[19] H.W. Hammer and R.J. Furnstahl, Nucl. Phys. A678, 277 (2000).

[20] A.L. Fetter and J.D. Walecka, Quantum Theory of Many-Particle Systems, (McGraw-Hill, New York, 1971) chapt. 12.

[21] E.K.U. Gross, E. Runge and O. Heinonen, Many-Particle Theory, (IOP Publishing Ltd, Bristol, 1991) chapt. 22.

[22] N. Kaiser, Nucl. Phys. A860, 41 (2011).

[23] N. Kaiser, Eur. Phys. J. A48, 148 (2012).

[24] N. Kaiser, Phys. Rev. C91, 065201 (2015). 